\title{
The New Solitary Solutions to the Time-Fractional Coupled Jaulent-Miodek Equation
}

\author{
Guiping Shen $\left(\mathbb{D},{ }^{1}\right.$ Jalil Manafian $\mathbb{D}^{2,3}$ Syed Maqsood Zia ${ }^{2}{ }^{4},{ }^{4}$ Dinh Tran Ngoc Huy $\left(\mathbb{D},{ }^{5,6}\right.$ \\ and Trung-Hieu Le (iD ${ }^{7}$ \\ ${ }^{1}$ College of Science, Shaoyang University, Shaoyang 422000, Hunan, China \\ ${ }^{2}$ Department of Applied Mathematics, Faculty of Mathematical Sciences, University of Tabriz, Tabriz, Iran \\ ${ }^{3}$ Natural Sciences Faculty, Lankaran State University, 50, H. Aslanov Str., Lankaran, Azerbaijan \\ ${ }^{4}$ Department of Statics, Faculty of Physical Sciences, Shah Abdul Latif University Khairpour, Khairpour, Sindh, Pakistan \\ ${ }^{5}$ Banking University HCMC, Ho Chi Minh City, Vietnam \\ ${ }^{6}$ International University of Japan, Niigata, Japan \\ ${ }^{7}$ Dai Nam University, Hanoi, Vietnam \\ Correspondence should be addressed to Jalil Manafian; j_manafianheris@tabrizu.ac.ir
}

Received 1 June 2021; Revised 1 September 2021; Accepted 13 September 2021; Published 13 November 2021

Academic Editor: Rodica Luca

Copyright (c) 2021 Guiping Shen et al. This is an open access article distributed under the Creative Commons Attribution License, which permits unrestricted use, distribution, and reproduction in any medium, provided the original work is properly cited.

\begin{abstract}
Here, two applicable methods, namely, the $\tan (\theta / 2)$-expansion technique and modified $\exp (-\theta(\xi))$-expansion technique are being applied on the time-fractional coupled Jaulent-Miodek equation. Materials such as photovoltaic-photorefractive, polymer, and organic contain spatial solitons and optical nonlinearities, which can be identified by seeking from energydependent Schrödinger potential. Plentiful exact traveling wave solutions containing unknown values are constructed in the sense of trigonometric, hyperbolic, exponential, and rational functions. Different arbitrary constants acquired in the solutions help us to discuss the dynamical behavior of solutions. Moreover, the graphical representation of solutions is shown vigorously in order to visualize the behavior of the solutions acquired for the mentioned equation. We obtain some periodic, dark soliton, and singular-kink wave solutions which have considerably fortified the existing literature on the timefractional coupled Jaulent-Miodek equation. Via three-dimensional plot, density plot, and two-dimensional plot by utilizing Maple software, the physical properties of these waves are explained very well.
\end{abstract}

\section{Introduction}

The main idea of this article focused on how we can get the new exact and numerical solutions for time-fractional coupled Jaulent-Miodek equation by using the new analytical methods, that is to say, the $\tan (\theta / 2)$-expansion method and the modified $\exp (-\theta(\xi))$-expansion method. Several authors studied the various nonlinear models through two different methods which may be applied to recent results such as the Biswas-Milovic equation for Kerr law nonlinearity [1], the Kerr law and dual power law Schrödinger equations [2], the fractional Bogoyavlenskii equations with conformable derivative [3], the Vakhnenko-Parkes equation [4], the Caudrey-Dodd-Gibbon and Pochhammer-Chree equations [5], some nonlinear fractional physical model [6], the time-fractional Kuramoto-Sivashinsky equation [7], new double-chain model of DNA, and a diffusive predator-prey system [8].

Authors usually use the fractional differential equations to explain a few attractive physical phenomena. Many terms we 
use naturedly or randomly in daily life often have a fractional structure. Verbal and numerical expressions that we use when describing something, explaining an event, and commanding and in many other cases contain behavior physical of fractional equations with wide range of nonlinear physical phenomena in the vast areas of scientific disciplines which are explained by nonlinear evolution equations. Owing to the importance of studying this class of equations, several distinct techniques have been proposed such as the fractional Davey-Stewartson equation with power law nonlinearity [9], the Calogero-Bogoyavlenskii-Schiff equation in $(2+1)$ dimensions with time-fractional conformable derivative [10], the fractional mitigating Internet bottleneck with quadratic cubic nonlinearity [11], the density-dependent conformable fractional diffusion-reaction equation [12], the space-time fractional Klein-Gordon equation with symmetry analysis [13], and the space-time fractional advection-diffusion equation with convergence analysis [14].
The further analytical methods for the $(2+1)$-dimensional Jaulent-Miodek (JM) evolution equation have been well scrutinized by a lot of researchers containing the results such as the Hirota's bilinear method [15], the expfunction method [16], the symmetry reductions method [17], the homotopy perturbation method [18], the optimal hidden symmetries [19], and the related topics of the Jaulent-Miodek equation with various topics, e.g., the $\left(G^{\prime} / G\right)$ expansion method [20], the bifurcation and exact traveling wave solutions [21], the integrating factors method in an unbounded domain [22], the Adomian's decomposition method [23], the finite-band solution method [24], N-fold Darboux transformation method [25], the Hermite wavelets method [26], and the modified Riemann-Liouville derivative and exterior derivatives [27]. In the following, we take the nonlinear time-fractional coupled Jaulent-Miodek (FCJM) equation, firstly introduced by Jaulent and Miodek [27-29], that is,

$$
\begin{aligned}
& \frac{\partial^{\alpha} u}{\partial t^{\alpha}}+\frac{\partial^{3} u}{\partial x^{3}}+\frac{3}{2} v \frac{\partial^{3} v}{\partial x^{3}}+\frac{9}{2} \frac{\partial v}{\partial x} \frac{\partial^{2} v}{\partial x^{2}}-6 u v \frac{\partial v}{\partial x}-6 u \frac{\partial u}{\partial x}-\frac{3}{2} v^{2} \frac{\partial u}{\partial x}=0, \quad 0 \leq \alpha<1, \\
& \frac{\partial^{\alpha} v}{\partial t^{\alpha}}+\frac{\partial^{3} v}{\partial x^{3}}-6 u \frac{\partial v}{\partial x}-6 v \frac{\partial u}{\partial x}-\frac{15}{2} v^{2} \frac{\partial v}{\partial x}=0, \quad 0 \leq \alpha<1 .
\end{aligned}
$$

Gupta and Ray [30] used the wavelet method based on the Hermite wavelet expansion and found the numerical solution to a coupled system of nonlinear time-fractional Jaulent-Miodek equations. The Lie symmetry analysis has been performed on a coupled system of nonlinear timefractional Jaulent-Miodek equations associated with energydependent Schrödinger potential to find the exact solution using Erdelyi-Kober fractional derivatives [31]. Moreover, the authors of [32-34] obtained new exact solutions for some of PDEs using the Hirota bilinear method.
The foremost objective in this article is discovering some periodic, soliton, singular, and singular-kink solutions by using two methods, namely, $\tan (\theta / 2)$-expansion method and modified $\exp (-\theta(\xi))$-expansion method [35], for the time-fractional coupled JM equations. In [36], Boulkhemair et al. used geometrical variations of a state-constrained functional on star-shaped domains. Nachaoui et al. investigated parallel numerical computation of an analytical method for solving an inverse problem in [37]. Use the modified Riemann-Liouville derivative of order $\alpha$ [38] as follows:

$$
D_{t}^{\alpha} u(t)= \begin{cases}\frac{1}{\Gamma(1-\alpha)} \int_{0}^{t}(t-\vartheta)^{-\alpha}(u(\vartheta)-u(0)) \mathrm{d} \vartheta, & \text { if } 0<\alpha \leq 1, \\ {\left[u^{(n)}(t)\right]^{(\alpha-n)},} & \text { if } n \leq \alpha<n+1, n \geq 1,\end{cases}
$$

with the below relations [39-41] 


$$
\left\{\begin{array}{l}
D_{J}^{\alpha}(f(x) g(x))=\sum_{j=0}^{+\infty}\left(\begin{array}{l}
\alpha \\
j
\end{array}\right) f^{(j)}(x) g_{R-L}^{(\alpha-j)}(x)-\frac{f(0) g(0)}{x^{\alpha} \Gamma(1-\alpha)} \\
D_{J}^{\alpha}(f(g(x)))=\sum_{j=0}^{+\infty}\left(\begin{array}{c}
\alpha \\
j
\end{array}\right) \frac{x^{j-\alpha} j !}{\Gamma(j-\alpha+1)} \sum_{m=1}^{j} f^{(m)}(g) \sum \prod_{k=1}^{j} \frac{1}{P_{k} !}\left(\frac{g^{(k)}}{k !}\right)^{P_{k}}+\frac{f(g(x))-f(g(0))}{x^{\alpha} \Gamma(1-\alpha)} \\
D_{t}^{\alpha} t^{\gamma}=\frac{\Gamma(\gamma+1)}{\Gamma(1+\alpha-\gamma)} t^{\gamma-\alpha}, \quad \gamma>0,
\end{array}\right.
$$

where $\Gamma$ denotes the gamma function.

In numerous scientific and engineering problems, to find exact analytical solutions of nonlinear partial differential equations (NLPDEs) for understanding many physical phenomena has great significance. So, establishing explicit traveling wave solutions of many physical systems has attracted considerable attention in the last few decades. Fortunately, in the literature, most of these methods are based on the hypothesis that the exact solution can be expressed as finite expansion of a function and use the following methodology in general to determine it. The method is efficiently used for several kinds of differential equations with respect to subsidiary conditions, types, etc. It includes initial value problems, boundary value problem, partial and ordinary differential equations, linear and nonlinear equations, and also nonlinear stochastic system as well as deterministic differential equations. In contrast to previously defined methods, this method extends more solutions containing periodic, hyperbolic, rational, kink, singular, and singular-kink solutions. In the continuation, we utilize concepts of local fractional calculus and its properties in Section 2. By utilizing of the symbolic calculation and employing the used TEM and MEEM, we want to solve the CFJM system in Sections 3 and 4. As a consequence, we plot some periodic, soliton, singular, and singular-kink solutions in Section 5. At the end, some concluding remarks about the obtained results are given.

\section{Description of Local Fractional Calculus and Its Properties}

Definition 1 (see [42-44]). Assume that $\varphi(x)$ is defined throughout some interval containing $x_{0}$ and all points near $x_{0}$, then $\varphi(x)$ is said to be local fractional continuous at $x=x_{0}$, symbolized by $\lim _{x \longrightarrow x_{0}} \varphi(x)=\varphi\left(x_{0}\right)$; then to each positive number $\varepsilon$ and also to a positive constant $\Omega$ corresponding some positive $\varepsilon$, we have

$$
\left|\varphi(x)-\varphi\left(x_{0}\right)\right|<\Omega \varepsilon^{\beta}, \quad 0 \leq \beta<1,
$$

whenever $\left|x-x_{0}\right|<\rho, \rho>0$ and $\varepsilon, \rho \in \mathbb{R}$. Therefore, the function $\varphi(x)$ is called local fractional continuous on the $(a, b)$,

$$
\varphi(x) \in C_{\beta}(a, b), \quad 0 \leq \beta<1,
$$

in which $\beta$ is the fractal dimension.

Definition 2 (see [42-44]). A function $\varphi(x): \mathbb{R} \longrightarrow$ $-\mathbb{R}, X \mapsto \varphi(X)$ is called to be nondifferentiable function of exponent $\beta, 0<\beta \leq 1$ which satisfies Hölder function of exponent $\beta$, then for $x, y \in X$, one gets

$$
|\varphi(x)-\varphi(y)|<C|x-y|^{\beta} .
$$

Definition 3 (see [42-44]). A function $\varphi(x): \mathbb{R} \longrightarrow$ $-\mathbb{R}, X \mapsto \varphi(X)$ is said to be local fractional continuous of order $\beta, 0<\beta \leq 1$, or in short, $\beta$-local fractional continuous, when we have

$$
\varphi(x)-\varphi\left(x_{0}\right)=O\left(\left(x-x_{0}\right)^{\beta}\right),
$$

for any $x_{0} \in[a, b]$ and $0<\beta \leq 1$.

Definition 4 (see [42-44]). A function $\varphi(x)$ is said to be in the space $C_{\beta}[a, b]$ if and only if it can be expressed as

$$
\varphi(x)-\varphi\left(x_{0}\right)=O\left(\left(x-x_{0}\right)^{\beta}\right),
$$

for any $x_{0} \in[a, b]$ and $0<\beta \leq 1$.

Definition 5 (see [42-44]). Get $\varphi(x) \in C_{\beta}(a, b)$. Hence, the local fractional derivative of $\varphi(x)$ having order $\beta, 0<\beta \leq 1$ at $x=x_{0}$ is defined as

$$
\varphi^{(\beta)}(x)=\left.\frac{\mathrm{d}^{\beta} \varphi(x)}{\mathrm{d} x^{\beta}}\right|_{x=x_{0}}=\lim _{x \longrightarrow x 0} \frac{\Delta^{\beta}\left(\varphi(x)-\varphi\left(x_{0}\right)\right)}{\left(x-x_{0}\right)^{\beta}},
$$


where $\Delta^{\beta}\left(\varphi(x)-\varphi\left(x_{0}\right)\right) \approx \Gamma(\beta+1) \Delta\left(\varphi(x)-\varphi\left(x_{0}\right)\right)$.

Remark 1 (see $[42,4,45,46]$ ). More important properties of the local fractional derivative are as follows:

$$
\left\{\begin{array}{l}
\text { (i) } \frac{\mathrm{d}^{\beta} x^{k \beta}}{\mathrm{d} x^{\beta}}=\frac{\Gamma(1+k \beta)}{\Gamma(1+(k-1) \beta)} x^{(k-1) \beta}, \\
\text { (ii) } \frac{\mathrm{d}^{\beta} E_{\beta}\left(\kappa x^{\beta}\right)}{\mathrm{d} x^{\beta}}=\kappa E_{\beta}\left(\kappa x^{\beta}\right) .
\end{array}\right.
$$

Remark 2 (see [42-47]). Moreover, the chain rule of the local fractional derivative is in the following:

$$
\begin{aligned}
\frac{\mathrm{d}^{\beta} \varphi(x)}{\mathrm{d} x^{\beta}} & =\Lambda^{(1)}(\theta(x)) \theta^{(\beta)}(x), \\
\varphi(x) & =\Lambda(\theta(x)),
\end{aligned}
$$

when $\Lambda^{(1)}(\theta(x))$ and $\theta^{(\beta)}(x)$ exist.

\section{Summarization of the $\tan (\boldsymbol{\theta}(\xi) / 2)$-Expansion Method}

In this section, we will describe the main steps of the $\tan (\theta(\xi) / 2)$-expansion method for finding traveling wave solutions of the time-fractional coupled Jaulent-Miodek equation in order to furnish its exact solutions:

Step 1. Given a nonlinear physical model governed by partial differential equation,

$$
\mathscr{P}\left(u, u_{x}, u_{x x}, u_{x x x}, \ldots, D_{t}^{\alpha} u, D_{t}^{2 \alpha} u, \ldots\right)=0,
$$

where fractional derivative is explained in local sense and $\mathscr{P}$ is a polynomial in $u(x, t)$ and its different partial derivatives can be converted into an ordinary differential equation (ODE),

$$
\mathscr{M}\left(u, r u^{\prime}, r^{2} u^{\prime \prime}, r^{3} u^{\prime \prime \prime}, \ldots, k u^{\prime}, \ldots\right)=0,
$$

by utilizing the suitable fractional complex change

$$
\begin{aligned}
u(x, t) & =u(\xi), \\
\xi & =r x+\frac{k}{\Gamma(\alpha+1)} t^{\alpha},
\end{aligned}
$$

where $r$ and $k$ are the unknown values which would be computed subsequently. Also, the chain rule [44] is utilized as

$$
\begin{aligned}
& D_{t}^{\alpha} u=\sigma_{t} u_{\xi} D_{t}^{\alpha} \xi, \\
& D_{x}^{\alpha} u=\sigma_{x} u_{\xi} D_{x}^{\alpha} \xi,
\end{aligned}
$$

where $\sigma_{t}$ and $\sigma_{x}$ are the fractal indexes $[43,48]$, without loss of generality, consider $\sigma_{t}=\sigma_{x}=\kappa$, in which $\kappa$ is a constant.

Step 2. Suppose that the solution of ODE (13) can be expressed by a polynomial in $\tan ^{k}(\theta(\xi) / 2)$ as follows:

$$
\begin{aligned}
& u(\xi)=S_{1}(\theta)=\sum_{k=0}^{m} A_{k} \tan ^{k}\left(\frac{\theta}{2}\right), \\
& v(\xi)=S_{2}(\theta)=\sum_{k=0}^{n} B_{k} \tan ^{k}\left(\frac{\theta}{2}\right),
\end{aligned}
$$

where $A_{k}(0 \leq k \leq m)$ and $B_{k}(1 \leq k \leq n)$ are constants to be found such that $A_{m} \neq 0, B_{n} \neq 0$ and $\theta=\theta(\xi)$ satisfies the following ordinary differential equation:

$$
\theta^{\prime}=k_{1} \sin (\theta)+k_{2} \cos (\theta)+k_{3} .
$$

We will take the below particular solutions of equation (17):

Family 1: if $\Delta=k_{1}^{2}+k_{2}^{2}-k_{3}^{2}<0$ and $k_{2}-k_{3} \neq 0$, afterwards $\theta(\xi)=2 \tan ^{-1}\left[\left(k_{1} /\left(k_{2}-k_{3}\right)\right)-\left(\sqrt{-\Delta} /\left(k_{2}-\right.\right.\right.$ $\left.\left.\left.k_{3}\right)\right) \tan ((\sqrt{-\Delta} / 2) \bar{\xi})\right]$

Family 2 : if $\Delta=k_{1}^{2}+k_{2}^{2}-k_{3}^{2}>0$ and $k_{2}-k_{3} \neq 0$, afterwards

$\theta(\xi)=2 \tan ^{-1}\left[\left(k_{1} /\left(k_{2}-k_{3}\right)\right)+\left(\sqrt{\Delta} /\left(k_{2}-k_{3}\right)\right) \tanh \right.$ $((\sqrt{\Delta} / 2) \bar{\xi})]$

Family 3: if $\Delta=k_{1}^{2}+k_{2}^{2}-k_{3}^{2}>0, k_{2} \neq 0$, and $k_{3}=0$, afterwards

$\theta(\xi)=2 \tan ^{-1}\left[\left(k_{1} / k_{2}\right)+\left(\sqrt{k_{2}^{2}+k_{1}^{2}} / k_{2}\right) \tanh \right.$

$\left.\left(\left(\sqrt{k_{2}^{2}+k_{1}^{2}} / 2\right) \bar{\xi}\right)\right]$

Family 4: if $\Delta=k_{1}^{2}+k_{2}^{2}-k_{3}^{2}<0, k_{3} \neq 0$, and $k_{2}=0$, afterwards

$\theta(\xi)=2 \tan ^{-1}\left[-\left(k_{1} / k_{3}\right)+\left(\sqrt{k_{3}^{2}+k_{1}^{2}} / k_{3}\right) \tan \right.$

$\left.\left(\left(\sqrt{k_{3}^{2}+k_{1}^{2}} / 2\right) \bar{\xi}\right)\right]$

Family 5: if $\Delta=k_{1}^{2}+k_{2}^{2}-k_{3}^{2}>0, k_{2}-k_{3} \neq 0$, and $k_{1}=0$, afterwards $\theta(\xi)=2 \tan ^{-1}\left[\sqrt{\left(k_{2}+k_{3}\right) /\left(k_{2}-k_{3}\right)}\right.$ $\left.\tanh \left(\sqrt{k_{2}^{2}-k_{3}^{2}} / 2 \bar{\xi}\right)\right]$.

Family 6: if $k_{1}=0$ and $k_{3}=0$, afterwards $\theta(\xi)=\tan ^{-1}\left[\left(\left(e^{2 k_{2} \xi}-1\right) /\left(e^{2 k_{2} \bar{\xi}}+1\right)\right),\left(2 e^{k_{2} \bar{\xi}} /\left(e^{2 k_{2} \bar{\xi}}+\right.\right.\right.$

1))]

Family 7: if $k_{2}=0$ and $k_{3}=0$, afterwards $\theta(\xi)=\tan ^{-1}$ $\left[\left(2 e^{k_{1}} \bar{\xi} /\left(e^{2 k_{1} \bar{\xi}^{2}}+1\right)\right),\left(e^{2 k_{1} \bar{\xi}}-1 /\left(e^{2 k_{1} \bar{\xi}}+1\right)\right)\right]$

Family 8: if $k_{1}^{2}+k_{2}^{2}=k_{3}^{2}$, afterwards $\theta(\xi)=2 \tan ^{-1}$ $\left[\left(k_{1} \bar{\xi}+2\right) /\left(k_{2}-k_{3}\right) \bar{\xi}\right]$

Family 9: If $k_{1}=k_{2}=k_{3}=k k_{1}$, afterwards $\theta(\xi)=$ $2 \tan ^{-1}\left[e^{k k_{1} \bar{\xi}}-1\right]$

Family 10: if $k_{1}=k_{3}=k k_{1}$ and $k_{2}=-k k_{1}$, afterwards $\theta(\xi)=-2 \tan ^{-1}\left[e^{k k_{1} \xi} /\left(-1+e^{k k_{1} \bar{\xi}}\right)\right]$

Family 11: if $k_{3}=k_{1}$, afterwards $\theta(\xi)=-2 \tan ^{-1}$ $\left[\left(\left(k_{1}+k_{2}\right) e^{k_{2} \xi}-1\right) /\left(k_{1}-k_{2}\right) e^{k_{2} \xi}-1\right]$ 
Family 12: if $k_{1}=k_{3}$, afterwards $\theta(\xi)=2 \tan ^{-1}$ $\left[\left(\left(k_{2}+k_{3}\right) e^{k_{2} \bar{\xi}}+1\right) /\left(\left(k_{2}-k_{3}\right) e^{k_{2} \bar{\xi}}-1\right)\right]$

Family 13: if $k_{3}=-k_{1}$, afterwards $\theta(\xi)=2 \tan ^{-1}$ $\left[\left(e^{k_{2} \bar{\xi}}+k_{2}-k_{1}\right) /\left(e^{k_{2} \bar{\xi}}-k_{2}-k_{1}\right)\right]$

Family 14: if $k_{2}=-k_{3}$, afterwards $\theta(\xi)=2 \tan ^{-1}$ $\left[k_{1} e^{k_{1} \xi} /\left(1-k_{3} e^{k_{1} \xi}\right)\right]$

Family 15: if $k_{2}=0$ and $k_{1}=k_{3}$, afterwards $\theta(\xi)=$ $-2 \tan ^{-1}\left[\left(k_{3} \bar{\xi}+2\right) / k_{3} \bar{\xi}\right]$

Family 16: if $k_{1}=0$ and $k_{2}=k_{3}$, afterwards $\theta(\xi)=$ $2 \tan ^{-1}\left[k_{3} \bar{\xi}\right]$

Family 17: if $k_{1}=0$ and $k_{2}=-k_{3}$, afterwards $\theta(\xi)=-2 \tan ^{-1}\left[1 / k_{3} \bar{\xi}\right]$

Family 18: if $k_{1}=0$ and $k_{2}=0$, afterwards $\theta(\xi)=$ $k_{3} \xi+C$

Family 19: if $k_{2}=k_{3}$, afterwards $\theta(\xi)=2 \tan ^{-1}\left[\left(e^{k_{1} \bar{\xi}}-\right.\right.$ $\left.\left.k_{3}\right) / k_{1}\right]$ where $A_{0}, A_{k}, B_{k}(k=1,2, \ldots, m), k_{1}, k_{2}$, and $k_{3}$ are free constants to be determined later. To determine the positive integers $m$ and $n$, it, usually, can be accomplished by the nonlinear terms of highest- order in equation (13) with the highest-order linear terms. Substituting equations (16) and (17) into equation (13) yields an equation of powers of $\tan (\theta / 2)^{k}$.

Step 3. With $N$ determined, we then collect all coefficients of powers of $\tan (\theta / 2)^{k}, \cot (\theta / 2)^{k}(k=0,1,2, \ldots)$ in the resulting equation where these coefficients have to vanish. This will give a system of algebraic equations involving the parameters

$A_{0}, A_{i}(i=1,2, \ldots, m), B_{i}(i=1,2, \ldots, n), k_{1}, k_{2}$, and $k_{3}$.

3.1. Periodic, Soliton, Singular, and Kink-Singular Solutions of the FCJM Equation by TEM. Take the fractional CJM equation as

$$
\begin{aligned}
& \frac{\partial^{\alpha} u}{\partial t^{\alpha}}+\frac{\partial^{3} u}{\partial x^{3}}+\frac{3}{2} v \frac{\partial^{3} v}{\partial x^{3}}+\frac{9}{2} \frac{\partial v}{\partial x} \frac{\partial^{2} v}{\partial x^{2}}-6 u v \frac{\partial v}{\partial x}-6 u \frac{\partial u}{\partial x}-\frac{3}{2} v^{2} \frac{\partial u}{\partial x}=0 \\
& \frac{\partial^{\alpha} v}{\partial t^{\alpha}}+\frac{\partial^{3} v}{\partial x^{3}}-6 u \frac{\partial v}{\partial x}-6 v \frac{\partial u}{\partial x}-\frac{15}{2} v^{2} \frac{\partial v}{\partial x}=0
\end{aligned}
$$

By using the transformation (14), equation (18) will be reduced to the following ODE:

$$
\begin{aligned}
& k u^{\prime}+r^{3} u^{\prime \prime \prime}+\frac{3}{2} r^{3} v v^{\prime \prime \prime}+\frac{9}{2} r^{3} v^{\prime} v^{\prime \prime}-6 r u v v^{\prime}-6 r u u^{\prime}-\frac{3}{2} r v^{2} u^{\prime}=0, \\
& k v^{\prime}+r^{3} v^{\prime \prime \prime}-6 r u v^{\prime}-6 r v u^{\prime}-\frac{15}{2} r v^{2} v^{\prime}=0 .
\end{aligned}
$$

Balancing $u^{\prime \prime \prime}$ with $v^{2} u^{\prime}$ and $v^{\prime \prime \prime}$ with $v^{2} v^{\prime}$ in equation (19) gives $m=2$ and $n=1$. We substitute the values of $m=$ 2 and $n=1$ in (16). To find the periodic waves of the fractional CJM equations, we would like to start from an ansatz as follows:

$$
\begin{aligned}
& u(\xi)=A_{0}+A_{1} \tan \left(\frac{\theta(\xi)}{2}\right)+A_{2} \tan \left(\frac{\theta(\xi)}{2}\right)^{2}, \\
& v(\xi)=B_{0}+B_{1} \tan \left(\frac{\theta(\xi)}{2}\right),
\end{aligned}
$$


where $A_{0}, A_{1}, A_{2}, B_{0}$, and $B_{1}$ are free parameters. Inserting (20) into equation (19) and then collecting the coefficients in the front of the various functions including $\tan (\theta(\xi) / 2)$ and $\cot (\theta(\xi) / 2)$ give the following:

$$
\begin{aligned}
& \left(\frac{1}{8}(-b+c)\left(18 b^{2} r^{3} B_{1}^{2}-36 b c r^{3} B_{1}^{2}+18 c^{2} r^{3} B_{1}^{2}+24 b^{2} r^{3} A_{2}-48 b c r^{3} A_{2}+24 c^{2} r^{3} A_{2}-36 r A_{2} B_{1}^{2}-48 r A_{2}^{2}\right)=0,\right. \\
& \frac{1}{4} a\left(18 b^{2} r^{3} B_{1}^{2}-36 b c r^{3} B_{1}^{2}+18 c^{2} r^{3} B_{1}^{2}+24 b^{2} r^{3} A_{2}-48 b c r^{3} A_{2}+24 c^{2} r^{3} A_{2}-36 r A_{2} B_{1}^{2}-48 r A_{2}^{2}\right) \\
& +\frac{1}{8}(-b+c)\left(45 a c r^{3} B_{1}^{2}-45 a b r^{3} B_{1}^{2}+9 b^{2} r^{3} B_{0} B_{1}-18 b c r^{3} B_{0} B_{1}+9 c^{2} r^{3} B_{0} B_{1}-60 a b r^{3} A_{2}+60 a c r^{3} A_{2}\right. \\
& \left.+6 b^{2} r^{3} A_{1}-12 b c r^{3} A_{1}+6 c^{2} r^{3} A_{1}-30 r A_{1} B_{1}^{2}-48 r A_{2} B_{0} B_{1}-72 r A_{1} A_{2}\right)=0, \\
& \frac{1}{8}(b+c)\left(18 b^{2} r^{3} B_{1}^{2}-36 b c r^{3} B_{1}^{2}+18 c^{2} r^{3} B_{1}^{2}+24 b^{2} r^{3} A_{2}-48 b c r^{3} A_{2}+24 c^{2} r^{3} A_{2}-36 r A_{2} B_{1}^{2}-48 r A_{2}^{2}\right)+\frac{1}{4} a\left(-45 a b r^{3} B_{1}^{2}\right. \\
& +45 a c r^{3} B_{1}^{2}+9 b^{2} r^{3} B_{0} B_{1}-18 b c r^{3} B_{0} B_{1}+9 c^{2} r^{3} B_{0} B_{1}-60 a b r^{3} A_{2}+60 a c r^{3} A_{2}+6 b^{2} r^{3} A_{1}-12 b c r^{3} A_{1} \\
& \left.+6 c^{2} r^{3} A_{1}-30 r A_{1} B_{1}^{2}-48 r A_{2} B_{0} B_{1}-72 r A_{1} A_{2}\right)+\frac{1}{8}(c-b)\left(\left(24 a^{2} r^{3}+12 c^{2} r^{3}-12 b^{2} r^{3}\right) B_{1}^{2}\right. \\
& +18 a r^{3} B_{0} B_{1}(b-c)+32 a^{2} r^{3} A_{2}-12 a b r^{3} A_{1}+12 a c r^{3} A_{1}-16 b^{2} r^{3} A_{2}+16 c^{2} r^{3} A_{2}-24 r A_{0} \\
& \left.B_{1}^{2}-36 r A_{1} B_{0} B_{1}-12 r A_{2} B_{0}^{2}-48 r A_{0} A_{2}-24 r A_{1}^{2}+8 k A_{2}\right)=0, \\
& \frac{1}{8}(b+c)\left(-45 a b r^{3} B_{1}^{2} 45 a c r^{3} B_{1}^{2}+9 b^{2} r^{3} B_{0} B_{1}-18 b c r^{3} B_{0} B_{1}+9 c^{2} r^{3} B_{0} B_{1}-60 a b r^{3} A_{2}+60 a c r^{3} A_{2}+6 b^{2} r^{3}\right. \\
& \left.A_{1}-12 b c r^{3} A_{1}+6 c^{2} r^{3} A_{1}-30 r A_{1} B_{1}^{2}-48 r A_{2} B_{0} B_{1}-72 r A_{1} A_{2}\right) \\
& +\frac{1}{4} a\left(24 a^{2} r^{3} B_{1}^{2}-18 a b r^{3} B_{0} B_{1}+18 a c r^{3} B_{0} B_{1}\right. \\
& -12 b^{2} r^{3} B_{1}^{2}+12 c^{2} r^{3} B_{1}^{2}+32 a^{2} r^{3} A_{2}-12 a b r^{3} A_{1}+12 a c r^{3} A_{1}-16 b^{2} r^{3} A_{2}+16 c^{2} r^{3} A_{2}-24 r A_{0} B_{1}^{2} \\
& \left.-36 r A_{1} B_{0} B_{1}-12 r A_{2} B_{0}^{2}-48 r A_{0} A_{2}-24 r A_{1}^{2}+8 k A_{2}\right)+\frac{1}{8}(-b+c)\left(6 a^{2} r^{3} B_{0} B_{1}+9 a b r^{3} B_{1}^{2}+9 a c r^{3} B_{1}^{2}\right. \\
& -3 b^{2} r^{3} B_{0} B_{1}+3 c^{2} r^{3} B_{0} B_{1}+4 a^{2} r^{3} A_{1}+12 a b r^{3} A_{2}+12 a c r^{3} A_{2}-2 b^{2} r^{3} A_{1}+2 c^{2} r^{3} A_{1}-24 r A_{0} B_{0} B_{1}-6 r A_{1} B_{0}^{2} \\
& \left.-24 r A_{0} A_{1}+4 k A_{1}\right)=0 \\
& \frac{1}{8}(b+c)\left(24 a^{2} r^{3} B_{1}^{2}-18 a b r^{3} B_{0} B_{1}+18 a c r^{3} B_{0} B_{1}-12 b^{2} r^{3} B_{1}^{2}+12 c^{2} r^{3} B_{1}^{2}+32 a^{2} r^{3} A_{2}\right. \\
& -12 a b r^{3} A_{1}+12 a c r^{3} A_{1}-16 b^{2} r^{3} A_{2}+16 c^{2} r^{3} A_{2}-24 r A_{0} B_{1}^{2}-36 r A_{1} B_{0} B_{1}-12 r A_{2} B_{0}^{2}-48 r A_{0} A_{2} \\
& \left.-24 r A_{1}^{2}+8 k A_{2}\right)+\frac{1}{4} a\left(6 a^{2} r^{3} B_{0} B_{1}+9 a b r^{3} B_{1}^{2}+9 a c r^{3} B_{1}^{2}\right. \\
& -3 b^{2} r^{3} B_{0} B_{1}+3 c^{2} r^{3} B_{0} B_{1}+4 a^{2} r^{3} A_{1}+12 a b r^{3} A_{2}+12 a c r^{3} A_{2} \\
& \left.-2 b^{2} r^{3} A_{1}+2 c^{2} r^{3} A_{1}-24 r A_{0} B_{0} B_{1}-6 r A_{1} B_{0}^{2}-24 r A_{0} A_{1}+4 k A_{1}\right) \frac{1}{8}(b+c)\left(24 a^{2} r^{3} B_{1}^{2}-18 a b r^{3} B_{0} B_{1}\right. \\
& +18 a c r^{3} B_{0} B_{1}-12 b^{2} r^{3} B_{1}^{2}+12 c^{2} r^{3} B_{1}^{2}+32 a^{2} r^{3} A_{2}-12 a b r^{3} A_{1}+12 a c r^{3} A_{1}-16 b^{2} r^{3} A_{2}+16 c^{2} r^{3} A_{2} \\
& \left.-24 r A_{0} B_{1}^{2}-36 r A_{1} B_{0} B_{1}-12 r A_{2} B_{0}^{2}-48 r A_{0} A_{2}-24 r A_{1}^{2}+8 k A_{2}\right)+\frac{1}{4} a\left(6 a^{2} r^{3} B_{0} B_{1}+9 a b r^{3} B_{1}^{2}\right. \\
& +9 a c r^{3} B_{1}^{2}-3 b^{2} r^{3} B_{0} B_{1}+3 c^{2} r^{3} B_{0} B_{1}+4 a^{2} r^{3} A_{1}+12 a b r^{3} A_{2}+12 a c r^{3} A_{2}-2 b^{2} r^{3} A_{1} \\
& \left.+2 c^{2} r^{3} A_{1}-24 r A_{0} B_{0} B_{1}-6 r A_{1} B_{0}^{2}-24 r A_{0} A_{1}+4 k A_{1}\right)=0,
\end{aligned}
$$




$$
\left\{\begin{array}{l}
\frac{1}{8}(b+c)\left(6 a^{2} r^{3} B_{0} B_{1}+9 a b r^{3} B_{1}^{2}+9 a c r^{3} B_{1}^{2}-3 b^{2} r^{3} B_{0} B_{1}+3 c^{2} r^{3} B_{0} B_{1}\right. \\
\left.+4 a^{2} r^{3} A_{1}+12 a b r^{3} A_{2}+12 a c r^{3} A_{2}-2 b^{2} r^{3} A_{1}+2 c^{2} r^{3} A_{1}-24 r A_{0} B_{0} B_{1}-6 r A_{1} B_{0}^{2}-24 r A_{0} A_{1}+4 k A_{1}\right)=0 \\
\frac{1}{4}(-b+c)\left(3 b^{2} r^{3} B_{1}-6 b c r^{3} B_{1}+3 c^{2} r^{3} B_{1}-15 r B_{1}^{3}-36 r A_{2} B_{1}\right)=0 \\
\frac{1}{2} a\left(3 b^{2} r^{3} B_{1}-6 b c r^{3} B_{1}+3 c^{2} r^{3} B_{1}-15 r B_{1}^{3}-36 r A_{2} B_{1}\right)+\frac{1}{4}(-b+c) \\
\left(-6 a b r^{3} B_{1}+6 a c r^{3} B_{1}-30 r B_{0} B_{1}^{2}-24 r A_{1} B_{1}-24 r A_{2} B_{0}\right)=0 \\
\frac{1}{4}(b+c)\left(3 b^{2} r^{3} B_{1}-6 b c r^{3} B_{1}+3 c^{2} r^{3} B_{1}-15 r B_{1}^{3}-36 r A_{2} B_{1}\right) \\
+\frac{1}{2} a\left(-6 a b r^{3} B_{1}+6 a c r^{3} B_{1}-30 r B_{0} B_{1}^{2}-24 r A_{1} B_{1}-24 r A_{2} B_{0}\right) \\
+\frac{1}{4}(-b+c)\left(2 a^{2} r^{3} B_{1}-b^{2} r^{3} B_{1}+c^{2} r^{3} B_{1}-15 r B_{0}^{2} B_{1}-12 r A_{0} B_{1}-12 r A_{1} B_{0}+2 k B_{1}\right)=0 \\
\frac{1}{4}(b+c)\left(-6 a b r^{3} B_{1}+6 a c r^{3} B_{1}-30 r B_{0} B_{1}^{2}-24 r A_{1} B_{1}-24 r A_{2} B_{0}\right)+ \\
\frac{1}{2} a\left(2 a^{2} r^{3} B_{1}-b^{2} r^{3} B_{1}+c^{2} r^{3} B_{1}-15 r B_{0}^{2} B_{1}-12 r A_{0} B_{1}-12 r A_{1} B_{0}+2 k B_{1}\right)=0 \\
\frac{1}{4}(b+c)\left(2 a^{2} r^{3} B_{1}-b^{2} r^{3} B_{1}+c^{2} r^{3} B_{1}-15 r B_{0}^{2} B_{1}-12 r A_{0} B_{1}-12 r A_{1} B_{0}+2 k B_{1}\right)=0 \\
\end{array}\right.
$$

By solving the nonlinear algebraic system of equations can conclude the following results:

Set I:

$$
\left\{\begin{array}{l}
k=-\frac{1}{4} r^{3}\left(4 k_{1}^{2}+k_{2}^{2}-k_{3}^{2}\right), \\
r=r \\
A_{0}=-\frac{1}{8}\left(k_{2}^{2}-k_{3}^{2}\right) r^{2} \\
A_{1}=-\frac{1}{4} r^{2} k_{1}\left(k_{2}-k_{3}\right) \\
A_{2}=\frac{3}{16} r^{2}\left(k_{2}-k_{3}\right)^{2} \\
B_{0}=0 \\
B_{1}=\frac{1}{2} r\left(k_{2}-k_{3}\right) \sqrt{-1}
\end{array}\right.
$$

Putting (22) into (20), we obtain a periodic solution of equation (18) by utilizing Family 1 as follows:

$$
\begin{aligned}
u_{1}= & -\frac{1}{8}\left(k_{2}^{2}-k_{3}^{2}\right) r^{2}-\frac{1}{4} r^{2} k_{1}\left(k_{2}-k_{3}\right) \\
& {\left[\frac{k_{1}}{k_{2}-k_{3}}-\frac{\sqrt{-\Delta}}{k_{2}-k_{3}} \tan \left(\frac{\sqrt{-\Delta}}{2} \bar{\xi}\right)\right]+} \\
& \cdot \frac{3}{16} r^{2}\left(k_{2}-k_{3}\right)^{2}\left[\frac{k_{1}}{k_{2}-k_{3}}-\frac{\sqrt{-\Delta}}{k_{2}-k_{3}} \tan \left(\frac{\sqrt{-\Delta}}{2} \bar{\xi}\right)\right]^{2},
\end{aligned}
$$

$$
v_{1}=\frac{1}{2} r\left(k_{2}-k_{3}\right) \sqrt{-1}\left[\frac{k_{1}}{k_{2}-k_{3}}-\frac{\sqrt{-\Delta}}{k_{2}-k_{3}} \tan \left(\frac{\sqrt{-\Delta}}{2} \bar{\xi}\right)\right] \text {, }
$$

where $\Delta=k_{1}^{2}+k_{2}^{2}-k_{3}^{2}<0$ and $\bar{\xi}=r x-(1 / 4) r^{3}$ $\left(4 k_{1}^{2}+k_{2}^{2}-k_{3}^{2}\right)\left(t^{\alpha} / \Gamma(\alpha+1)\right)+C$.

Appending (22) into (20), we perceive a hyperbolic solution of equation (18) by utilizing Family 2 as follows: 


$$
\begin{aligned}
u_{2}= & -\frac{1}{8}\left(k_{2}^{2}-k_{3}^{2}\right) r^{2}-\frac{1}{4} r^{2} k_{1}\left(k_{2}-k_{3}\right)\left[\frac{k_{1}}{k_{2}-k_{3}}+\frac{\sqrt{\Delta}}{k_{2}-k_{3}} \tanh \left(\frac{\sqrt{\Delta}}{2} \bar{\xi}\right)\right]+ \\
& \frac{3}{16} r^{2}\left(k_{2}-k_{3}\right)^{2}\left[\frac{k_{1}}{k_{2}-k_{3}}+\frac{\sqrt{\Delta}}{k_{2}-k_{3}} \tanh \left(\frac{\sqrt{\Delta}}{2} \bar{\xi}\right)\right]^{2}, \\
v_{2}= & \frac{1}{2} r\left(k_{2}-k_{3}\right) \sqrt{-1}\left[\frac{k_{1}}{k_{2}-k_{3}}+\frac{\sqrt{\Delta}}{k_{2}-k_{3}} \tanh \left(\frac{\sqrt{\Delta}}{2} \bar{\xi}\right)\right],
\end{aligned}
$$

where $\Delta=k_{1}^{2}+k_{2}^{2}-k_{3}^{2}>0$ and $\bar{\xi}=r x-(1 / 4) r^{3}$ $\left(4 k_{1}^{2}+k_{2}^{2}-k_{3}^{2}\right)\left(t^{\alpha} / \Gamma(\alpha+1)\right)+C$.
Substituting (22) into (20), we perceive a hyperbolic solution of equation (18) by utilizing Family 8 as follows:

$$
\begin{aligned}
& u_{3}=-\frac{1}{8}\left(k_{2}^{2}-k_{3}^{2}\right) r^{2}-\frac{1}{4} r^{2} k_{1}\left(k_{2}-k_{3}\right)\left[\frac{k_{1} \bar{\xi}+2}{\left(k_{2}-k_{3}\right) \bar{\xi}}\right]+\frac{3}{16} r^{2}\left(k_{2}-k_{3}\right)^{2}\left[\frac{k_{1} \bar{\xi}+2}{\left(k_{2}-k_{3}\right) \bar{\xi}}\right]^{2}, \\
& v_{3}=\frac{1}{2} r\left(k_{2}-k_{3}\right) \sqrt{-1} \frac{3}{16} r^{2}\left(k_{2}-k_{3}\right)^{2}\left[\frac{k_{1} \bar{\xi}+2}{\left(k_{2}-k_{3}\right) \bar{\xi}}\right],
\end{aligned}
$$

where $k_{1}^{2}=k_{3}^{2}-k_{2}^{2} \quad$ and $\quad \bar{\xi}=r x-(3 / 3) r^{3} k_{1}^{2} \quad\left(t^{\alpha} /\right.$ $\Gamma(\alpha+1))+C$.
Inserting (22) into (20), we gain a hyperbolic solution of equation (18) by utilizing Family 12 as follows:

$$
\begin{aligned}
& u_{4}=-\frac{1}{8}\left(k_{2}^{2}-k_{3}^{2}\right) r^{2}-\frac{1}{4} r^{2} k_{3}\left(k_{2}-k_{3}\right)\left[\frac{\left(k_{2}+k_{3}\right) e^{k_{2} \bar{\xi}}+1}{\left(k_{2}-k_{3}\right) e^{k_{2} \bar{\xi}}-1}\right]+\frac{3}{16} r^{2}\left(k_{2}-k_{3}\right)^{2}\left[\frac{\left(k_{2}+k_{3}\right) e^{k_{2} \bar{\xi}}+1}{\left(k_{2}-k_{3}\right) e^{k_{2} \bar{\xi}}-1}\right]^{2}, \\
& v_{4}=\frac{1}{2} r\left(k_{2}-k_{3}\right) \sqrt{-1} \frac{3}{16} r^{2}\left(k_{2}-k_{3}\right)^{2}\left[\frac{\left(k_{2}+k_{3}\right) e^{k_{2} \bar{\xi}}+1}{\left(k_{2}-k_{3}\right) e^{k_{2} \bar{\xi}}-1}\right],
\end{aligned}
$$

where $\bar{\xi}=r x-(1 / 4) r^{3}\left(3 k_{3}^{2}+k_{2}^{2}\right)\left(t^{\alpha} / \Gamma(\alpha+1)\right)+C$.

Set II: 


$$
\left\{\begin{array}{l}
k=2 \frac{\left(4 k_{1}^{2} B_{1}^{2}+6 k_{1} k_{2} B_{0} B_{1}-6 k_{1} k_{3} B_{0} B_{1}+3 b^{2} B_{0}^{2}+k_{2}^{2} B_{1}^{2}-6 k_{2} k_{3} B_{0}^{2}+3 k_{3}^{2} B_{0}^{2}-k_{3}^{2} B_{1}^{2}\right) \sqrt{-1} B_{1}}{\left(k_{2}-k_{3}\right)^{3}} \\
r=\frac{2 B_{1} \sqrt{-1}}{k_{2}-k_{3}}, \\
A_{0}=-\frac{1}{4} \frac{k_{2} B_{0}^{2}-2 k_{2} B_{1}^{2}-k_{3} B_{0}^{2}-2 k_{3} B_{1}^{2}}{k_{2}-k_{3}}, \\
A_{1}=\frac{1}{2} \frac{\left(2 k_{1} B_{1}-k_{2} B_{0}+k_{3} B_{0}\right) B_{1}}{k_{2}-k_{3}}, \\
A_{2}=-\frac{3}{4} B_{1}^{2}, \\
B_{0}=B_{0}
\end{array}\right.
$$

Substituting (31) into (20), we perceive a periodic solution of equation (18) by utilizing Family 1 as follows:

$$
\begin{aligned}
u_{5}= & -\frac{1}{4} \frac{k_{2} B_{0}^{2}-2 k_{2} B_{1}^{2}-k_{3} B_{0}^{2}-2 k_{3} B_{1}^{2}}{k_{2}-k_{3}}+\frac{1}{2} \frac{\left(2 k_{1} B_{1}-k_{2} B_{0}+k_{3} B_{0}\right) B_{1}}{k_{2}-k_{3}}\left[\frac{k_{1}}{k_{2}-k_{3}}-\frac{\sqrt{-\Delta}}{k_{2}-k_{3}} \tan \left(\frac{\sqrt{-\Delta}}{2} \bar{\xi}\right)\right] \\
& -\frac{3}{4} B_{1}^{2}\left[\frac{k_{1}}{k_{2}-k_{3}}-\frac{\sqrt{-\Delta}}{k_{2}-k_{3}} \tan \left(\frac{\sqrt{-\Delta}}{2} \bar{\xi}\right)\right]^{2}, \\
v_{5}= & B_{0}+B_{1}\left[\frac{k_{1}}{k_{2}-k_{3}}-\frac{\sqrt{-\Delta}}{k_{2}-k_{3}} \tan \left(\frac{\sqrt{-\Delta}}{2} \bar{\xi}\right)\right],
\end{aligned}
$$

where $\quad \Delta=k_{1}^{2}+k_{2}^{2}-k_{3}^{2}<0 \quad$ and $\quad \bar{\xi}=\left(2 B_{1} \sqrt{-1} /\right.$ $\left.\left(k_{2}-k_{3}\right)\right) \quad x+2\left(\left(4 k_{1}^{2} B_{1}^{2}+6 k_{1} k_{2} B_{0} B_{1}-6 k_{1} k_{3} B_{0} B_{1}+\right.\right.$ $\left.3 k_{2}^{2} \quad B_{0}^{2}+k_{2}^{2} B_{1}^{2}-6 k_{2} k_{3} B_{0}^{2}+3 k_{3}^{2} B_{0}^{2}-k_{3}^{2} B_{1}^{2}\right) \sqrt{-1} B_{1} /$ $\left.\left(k_{2}-k_{3}\right)^{3}\right)\left(t^{\alpha} / \Gamma(\alpha+1)\right)+C$.
Appending (31) into (20), we gain a hyperbolic solution of equation (18) by utilizing Family 2 as follows:

$$
\begin{aligned}
u_{6}= & -\frac{1}{4} \frac{k_{2} B_{0}^{2}-2 k_{2} B_{1}^{2}-k_{3} B_{0}^{2}-2 k_{3} B_{1}^{2}}{k_{2}-k_{3}}+\frac{1}{2} \frac{\left(2 k_{1} B_{1}-k_{2} B_{0}+k_{3} B_{0}\right) B_{1}}{k_{2}-k_{3}}\left[\frac{k_{1}}{k_{2}-k_{3}}+\frac{\sqrt{\Delta}}{k_{2}-k_{3}} \tanh \left(\frac{\sqrt{\Delta}}{2} \bar{\xi}\right)\right] \\
& -\frac{3}{4} B_{1}^{2}\left[\frac{k_{1}}{k_{2}-k_{3}}+\frac{\sqrt{\Delta}}{k_{2}-k_{3}} \tanh \left(\frac{\sqrt{\Delta}}{2} \bar{\xi}\right)\right]^{2}, \\
v_{6}= & B_{0}+B_{1}\left[\frac{k_{1}}{k_{2}-k_{3}}+\frac{\sqrt{\Delta}}{k_{2}-k_{3}} \tanh \left(\frac{\sqrt{\Delta}}{2} \bar{\xi}\right)\right],
\end{aligned}
$$


where $\Delta=k_{1}^{2}+k_{2}^{2}-k_{3}^{2}>0$ and $\bar{\xi}=\left(2 B_{1} \sqrt{-1} / \quad\left(k_{2}-\right.\right.$ $\left.\left.k_{3}\right)\right) x+2\left(\left(4 k_{1}^{2} B_{1}^{2}+6 k_{1} k_{2} B_{0} B_{1}-6 k_{1} k_{3} B_{0} B_{1}+3 k_{2}^{2} B_{0}^{2}+\right.\right.$ $\left.\left.k_{2}^{2} B_{1}^{2}-6 k_{2} k_{3} B_{0}^{2}+3 k_{3}^{2} B_{0}^{2}-k_{3}^{2} B_{1}^{2}\right) \sqrt{-1} B_{1} / \quad\left(k_{2}-k_{3}\right)^{3}\right)$ $\left(t^{\alpha} / \Gamma(\alpha+1)\right)+C$.
Substituting (31) into (20), we perceive a hyperbolic solution of equation (18) by utilizing Family 8 as follows:

$$
\begin{aligned}
& u_{7}=-\frac{1}{4} \frac{k_{2} B_{0}^{2}-2 k_{2} B_{1}^{2}-k_{3} B_{0}^{2}-2 k_{3} B_{1}^{2}}{k_{2}-k_{3}}+\frac{1}{2} \frac{\left(2 k_{1} B_{1}-k_{2} B_{0}+k_{3} B_{0}\right) B_{1}}{k_{2}-k_{3}}\left[\frac{k_{1} \bar{\xi}+2}{\left(k_{2}-k_{3}\right) \bar{\xi}}\right]-\frac{3}{4} B_{1}^{2}\left[\frac{k_{1} \bar{\xi}+2}{\left(k_{2}-k_{3}\right) \bar{\xi}}\right]^{2}, \\
& v_{7}=B_{0}+B_{1}\left[\frac{k_{1} \bar{\xi}+2}{\left(k_{2}-k_{3}\right) \bar{\xi}}\right],
\end{aligned}
$$

where $k_{1}^{2}+k_{2}^{2}-k_{3}^{2}=0$ and $\bar{\xi}=\left(2 B_{1} \sqrt{-1} /\left(k_{2}-k_{3}\right)\right) x$ $+2\left(\left(4 k_{1}^{2} B_{1}^{2}+6 k_{1} k_{2} B_{0} B_{1}-6 k_{1} k_{3} B_{0} B_{1}+3 k_{2}^{2} B_{0}^{2}+k_{2}^{2} B_{1}^{2}\right.\right.$ $\left.\left.-6 k_{2} k_{3} B_{0}^{2}+3 k_{3}^{2} B_{0}^{2}-k_{3}^{2} B_{1}^{2}\right) \sqrt{-1} B_{1} /\left(k_{2}-k_{3}\right)^{3}\right)\left(t^{\alpha} / \Gamma(\alpha\right.$ $+1))+C$.
Inserting (31) into (20), we receive a hyperbolic solution of equation (18) by utilizing Family 12 as follows:

$$
\begin{aligned}
u_{8}= & -\frac{1}{4} \frac{k_{2} B_{0}^{2}-2 k_{2} B_{1}^{2}-k_{3} B_{0}^{2}-2 k_{3} B_{1}^{2}}{k_{2}-k_{3}}+\frac{1}{2} \frac{\left(2 k_{3} B_{1}-k_{2} B_{0}+k_{3} B_{0}\right) B_{1}}{k_{2}-k_{3}}\left[\frac{\left(k_{2}+k_{3}\right) e^{k_{2} \bar{\xi}}+1}{\left(k_{2}-k_{3}\right) e^{k_{2} \bar{\xi}}-1}\right] \\
& -\frac{3}{4} B_{1}^{2}\left[\frac{\left(k_{2}+k_{3}\right) e^{k_{2} \bar{\xi}}+1}{\left(k_{2}-k_{3}\right) e^{k_{2} \bar{\xi}}-1}\right], \\
v_{8}= & B_{0}+B_{1}\left[\frac{\left(k_{2}+k_{3}\right) e^{k_{2} \bar{\xi}}+1}{\left(k_{2}-k_{3}\right) e^{k_{2} \bar{\xi}}-1}\right],
\end{aligned}
$$

where $k_{1}=k_{3}$ and $\bar{\xi}=\left(2 B_{1} \sqrt{-1} /\left(k_{2}-k_{3}\right)\right)$ $x+2\left(\left(3 k_{3}^{2} B_{1}^{2}+6 k_{3} k_{2} B_{0} B_{1}-6 k_{3}^{2} B_{0} B_{1}+3 k_{2}^{2} B_{0}^{2}+k_{2}^{2} B_{1}^{2}\right.\right.$ $\left.\left.-6 k_{2} k_{3} B_{0}^{2}+3 k_{3}^{2} B_{0}^{2}\right) \sqrt{-1} B_{1} / \quad\left(k_{2}-k_{3}\right)^{3}\right)\left(t^{\alpha} / \Gamma(\alpha+1)\right)$ $+C$.

Set III:

$$
\left\{\begin{array}{l}
k=-\frac{1}{4}\left(76 k_{1}^{2}+k_{2}^{2}-k_{3}^{2}\right) r^{3}, \\
r=r \\
A_{0}=\frac{1}{8} r^{2}\left(18 k_{1}^{2}-k_{2}^{2}+k_{3}^{2}\right), \\
A_{1}=-\left(k_{2}-k_{3}\right) a r^{2}, \\
A_{2}=\frac{3}{16} r^{2}\left(k_{2}-k_{3}\right)^{2}, \\
B_{0}=-3 k_{1} r \sqrt{-1}, \\
B_{1}=\frac{1}{2} r\left(k_{2}-k_{3}\right) \sqrt{-1} .
\end{array}\right.
$$

Inserting (36) into (20), we perceive a periodic solution of equation (18) by utilizing Family 1 as follows:

$$
\begin{aligned}
u_{9}= & \frac{1}{8} r^{2}\left(18 k_{1}^{2}-k_{2}^{2}+k_{3}^{2}\right)-\left(k_{2}-k_{3}\right) k_{1} r^{2} \\
& \cdot\left[\frac{k_{1}}{k_{2}-k_{3}}-\frac{\sqrt{-\Delta}}{k_{2}-k_{3}} \tan \left(\frac{\sqrt{-\Delta}}{2} \bar{\xi}\right)\right] \\
& +\frac{3}{16} r^{2}\left(k_{2}-k_{3}\right)^{2}\left[\frac{k_{1}}{k_{2}-k_{3}}-\frac{\sqrt{-\Delta}}{k_{2}-k_{3}} \tan \left(\frac{\sqrt{-\Delta}}{2} \bar{\xi}\right)\right]^{2}, \\
v_{9}= & -3 k_{1} r \sqrt{-1}+\frac{1}{2} r\left(k_{2}-k_{3}\right) \sqrt{-1} \\
& \cdot\left[\frac{k_{1}}{k_{2}-k_{3}}-\frac{\sqrt{-\Delta}}{k_{2}-k_{3}} \tan \left(\frac{\sqrt{-\Delta}}{2} \bar{\xi}\right)\right],
\end{aligned}
$$

where $\quad \Delta=k_{1}^{2}+k_{2}^{2}-k_{3}^{2}<0$ and $\bar{\xi}=r x-(1 / 4)$ $\left(76 k_{1}^{2}+k_{2}^{2}-k_{3}^{2}\right) r^{3}\left(t^{\alpha} / \Gamma(\alpha+1)\right)+C$.

Appending (36) into (20), we obtain a hyperbolic solution of equation (18) by utilizing Family 2 as follows: 


$$
\begin{aligned}
u_{10}= & \frac{1}{8} r^{2}\left(18 k_{1}^{2}-k_{2}^{2}+k_{3}^{2}\right)-\left(k_{2}-k_{3}\right) k_{1} r^{2}\left[\frac{k_{1}}{k_{2}-k_{3}}+\frac{\sqrt{\Delta}}{k_{2}-k_{3}} \tanh \left(\frac{\sqrt{\Delta}}{2} \bar{\xi}\right)\right] \\
& +\frac{3}{16} r^{2}\left(k_{2}-k_{3}\right)^{2}\left[\frac{k_{1}}{k_{2}-k_{3}}+\frac{\sqrt{\Delta}}{k_{2}-k_{3}} \tanh \left(\frac{\sqrt{\Delta}}{2} \bar{\xi}\right)\right]^{2}, \\
v_{10}= & -3 k_{1} r \sqrt{-1}+\frac{1}{2} r\left(k_{2}-k_{3}\right) \sqrt{-1}\left[\frac{k_{1}}{k_{2}-k_{3}}+\frac{\sqrt{\Delta}}{k_{2}-k_{3}} \tanh \left(\frac{\sqrt{\Delta}}{2} \bar{\xi}\right)\right],
\end{aligned}
$$

where $\quad \Delta=k_{1}^{2}+k_{2}^{2}-k_{3}^{2}<0$ and $\bar{\xi}=r x-(1 / 4)$ $\left(76 k_{1}^{2}+k_{2}^{2}-k_{3}^{2}\right) r^{3}\left(t^{\alpha} / \Gamma(\alpha+1)\right)+C$.
Inserting (36) into (20), we perceive a hyperbolic solution of equation (18) by utilizing Family 8 as follows:

$$
\begin{aligned}
& u_{11}=\frac{1}{8} r^{2}\left(18 k_{1}^{2}-k_{2}^{2}+k_{3}^{2}\right)-\left(k_{2}-k_{3}\right) k_{1} r^{2}\left[\frac{k_{1} \bar{\xi}+2}{\left(k_{2}-k_{3}\right) \bar{\xi}}\right]+\frac{3}{16} r^{2}\left(k_{2}-k_{3}\right)^{2}\left[\frac{k_{1} \bar{\xi}+2}{\left(k_{2}-k_{3}\right) \bar{\xi}}\right]^{2}, \\
& v_{11}=-3 k_{1} r \sqrt{-1}+\frac{1}{2} r\left(k_{2}-k_{3}\right) \sqrt{-1}\left[\frac{k_{1} \bar{\xi}+2}{\left(k_{2}-k_{3}\right) \bar{\xi}}\right],
\end{aligned}
$$

where $\quad k_{1}^{2}+k_{2}^{2}-k_{3}^{2}=0 \quad$ and $\quad \bar{\xi}=r x-(75 / 4)$ $k_{1}^{2} r^{3}\left(t^{\alpha} / \Gamma(\alpha+1)\right)+C$.

Appending (36) into (20), we gain a hyperbolic solution of equation (18) by utilizing Family 12 as follows:

$$
\begin{aligned}
& u_{12}=\frac{1}{8} r^{2}\left(19 k_{1}^{2}-k_{2}^{2}\right)-\left(k_{2}-k_{3}\right) k_{3} r^{2}\left[\frac{\left(k_{2}+k_{3}\right) e^{k_{2} \bar{\xi}}+1}{\left(k_{2}-k_{3}\right) e^{k_{2} \bar{\xi}}-1}\right]+\frac{3}{16} r^{2}\left(k_{2}-k_{3}\right)^{2}\left[\frac{\left(k_{2}+k_{3}\right) e^{k_{2} \bar{\xi}}+1}{\left(k_{2}-k_{3}\right) e^{k_{2} \bar{\xi}}-1}\right]^{2}, \\
& v_{12}=-3 k_{3} r \sqrt{-1}+\frac{1}{2} r\left(k_{2}-k_{3}\right) \sqrt{-1}\left[\frac{\left(k_{2}+k_{3}\right) e^{k_{2} \bar{\xi}}+1}{\left(k_{2}-k_{3}\right) e^{k_{2} \bar{\xi}}-1}\right],
\end{aligned}
$$

where $k_{1}=k_{3} \quad$ and $\quad \bar{\xi}=r x-(1 / 4)\left(75 k_{3}^{2}+k_{2}^{2}\right) r^{3}$ $\left(t^{\alpha} / \Gamma(\alpha+1)\right)+C$.

Set IV:

$$
\left\{\begin{array}{l}
k=-\left(k_{1}^{2}+k_{2}^{2}-k_{3}^{2}\right) r^{3}, \\
r=r \\
A_{0}=\frac{1}{4} r^{2}\left(k_{1}^{2}-k_{2}^{2}+k_{3}^{2}\right), \\
A_{1}=-\left(k_{2}-k_{3}\right) k_{1} r^{2} \\
A_{2}=\frac{1}{2} r^{2}\left(k_{2}-k_{3}\right)^{2} \\
B_{0}=-k_{1} r \sqrt{-1} \\
B_{1}=r\left(k_{2}-k_{3}\right) \sqrt{-1} .
\end{array}\right.
$$

Substituting (41) into (20), we perceive a periodic solution of equation (18) by utilizing Family 1 as follows:

$$
\begin{aligned}
u_{13}= & \frac{1}{4} r^{2}\left(k_{1}^{2}-k_{2}^{2}+k_{3}^{2}\right)-\left(k_{2}-k_{3}\right) k_{1} r^{2} \\
& \cdot\left[\frac{k_{1}}{k_{2}-k_{3}}-\frac{\sqrt{-\Delta}}{k_{2}-k_{3}} \tan \left(\frac{\sqrt{-\Delta}}{2} \bar{\xi}\right)\right] \\
& +\frac{1}{2} r^{2}\left(k_{2}-k_{3}\right)^{2}\left[\frac{k_{1}}{k_{2}-k_{3}}-\frac{\sqrt{-\Delta}}{k_{2}-k_{3}} \tan \left(\frac{\sqrt{-\Delta}}{2} \bar{\xi}\right)\right]^{2}, \\
v_{13}= & -k_{1} r \sqrt{-1}+r\left(k_{2}-k_{3}\right) \sqrt{-1} \\
& \cdot\left[\frac{k_{1}}{k_{2}-k_{3}}-\frac{\sqrt{-\Delta}}{k_{2}-k_{3}} \tan \left(\frac{\sqrt{-\Delta}}{2} \bar{\xi}\right)\right],
\end{aligned}
$$

where $\Delta=k_{1}^{2}+k_{2}^{2}-k_{3}^{2}<0$ and $\bar{\xi}=r x-\left(k_{1}^{2}+k_{2}^{2}-k_{3}^{2}\right) r^{3}$ $\left(t^{\alpha} / \Gamma(\alpha+1)\right)+C$.

Inserting (41) into (20), we gain a hyperbolic solution of equation (18) by utilizing Family 2 as follows: 


$$
\begin{aligned}
u_{14}= & \frac{1}{4} r^{2}\left(k_{1}^{2}-k_{2}^{2}+k_{3}^{2}\right)-\left(k_{2}-k_{3}\right) k_{1} r^{2}\left[\frac{k_{1}}{k_{2}-k_{3}}+\frac{\sqrt{\Delta}}{k_{2}-k_{3}} \tanh \left(\frac{\sqrt{\Delta}}{2} \bar{\xi}\right)\right] \\
& +\frac{1}{2} r^{2}\left(k_{2}-k_{3}\right)^{2}\left[\frac{k_{1}}{k_{2}-k_{3}}+\frac{\sqrt{\Delta}}{k_{2}-k_{3}} \tanh \left(\frac{\sqrt{\Delta}}{2} \bar{\xi}\right)\right]^{2}, \\
v_{14}= & -k_{1} r \sqrt{-1}+r\left(k_{2}-k_{3}\right) \sqrt{-1}\left[\frac{k_{1}}{k_{2}-k_{3}}+\frac{\sqrt{\Delta}}{k_{2}-k_{3}} \tanh \left(\frac{\sqrt{\Delta}}{2} \bar{\xi}\right)\right],
\end{aligned}
$$

where $\Delta=k_{1}^{2}+k_{2}^{2}-k_{3}^{2}<0$ and $\bar{\xi}=r x-\left(k_{1}^{2}+k_{2}^{2}-k_{3}^{2}\right) r^{3}$ $\left(t^{\alpha} / \Gamma(\alpha+1)\right)+C$.
Plugging (41) into (20), we perceive a hyperbolic solution of equation (18) by utilizing Family 8 as follows:

$$
\begin{aligned}
& u_{15}=\frac{1}{4} r^{2}\left(k_{1}^{2}-k_{2}^{2}+k_{3}^{2}\right)-\left(k_{2}-k_{3}\right) k_{1} r^{2}\left[\frac{k_{1} \bar{\xi}+2}{\left(k_{2}-k_{3}\right) \bar{\xi}}\right]+\frac{1}{2} r^{2}\left(k_{2}-k_{3}\right)^{2}\left[\frac{k_{1} \bar{\xi}+2}{\left(k_{2}-k_{3}\right) \bar{\xi}}\right]^{2}, \\
& v_{15}=-k_{1} r \sqrt{-1}+r\left(k_{2}-k_{3}\right) \sqrt{-1}\left[\frac{k_{1} \bar{\xi}+2}{\left(k_{2}-k_{3}\right) \bar{\xi}}\right],
\end{aligned}
$$

where $\Delta=k_{1}^{2}+k_{2}^{2}-k_{3}^{2} \longrightarrow 0$ and $\bar{\xi}=r x-(\Delta \longrightarrow 0) r^{3}$ $r^{3}\left(t^{\alpha} / \Gamma(\alpha+1)\right)+C$.
Inserting (41) into (20), we perceive a hyperbolic solution of equation (18) by utilizing Family 12 as follows:

$$
\begin{aligned}
& u_{16}=\frac{1}{4} r^{2}\left(2 k_{3}^{2}-k_{2}^{2}\right)-\left(k_{2}-k_{3}\right) k_{3} r^{2}\left[\frac{\left(k_{2}+k_{3}\right) e^{k_{2} \bar{\xi}}+1}{\left(k_{2}-k_{3}\right) e^{k_{2} \bar{\xi}}-1}\right]+\frac{1}{2} r^{2}\left(k_{2}-k_{3}\right)^{2}\left[\frac{\left(k_{2}+k_{3}\right) e^{k_{2} \bar{\xi}}+1}{\left(k_{2}-k_{3}\right) e^{k_{2} \bar{\xi}}-1}\right]^{2}, \\
& v_{16}=-k_{3} r \sqrt{-1}+r\left(k_{2}-k_{3}\right) \sqrt{-1}\left[\frac{\left(k_{2}+k_{3}\right) e^{k_{2} \bar{\xi}}+1}{\left(k_{2}-k_{3}\right) e^{k_{2} \bar{\xi}}-1}\right],
\end{aligned}
$$

where $k_{1}=k_{3}$ and $\bar{\xi}=r x-k_{2}^{2} r^{3}\left(t^{\alpha} / \Gamma(\alpha+1)\right)+C$.

\section{Summarization of the Modified $\exp (-\boldsymbol{\theta}(\boldsymbol{\xi}))$-Expansion Method}

In this section, we will describe the main steps of the modified $\exp (-\theta(\xi))$-expansion method for finding traveling wave solutions of NLPDEs to the time-fractional coupled Jaulent-Miodek equation in order to furnish its exact solutions:
Step 1. Given a nonlinear physical model governed by partial differential equation of fractional order is expressed as

$$
p\left(u, u_{x}, u_{x x}, u_{x x x}, \ldots, D_{t}^{\alpha} u, D_{t}^{2 \alpha} u, \ldots\right)=0,
$$

the above equation can be converted into an ordinary differential equation (ODE),

$$
M\left(u, r u^{\prime}, r^{2} u^{\prime \prime}, r^{3} u^{\prime \prime \prime}, \ldots, k u^{\prime}, \ldots\right)=0,
$$


by utilizing the suitable fractional complex transform

$$
\begin{aligned}
u(x, t) & =u(\xi), \\
\xi & =r x+\frac{k}{\Gamma(\alpha+1)} t^{\alpha},
\end{aligned}
$$

where $r$ and $k$ are free parameters which would be computed subsequently. Also, the chain rule [44] is used as

$$
\begin{aligned}
& D_{t}^{\alpha} u=\sigma_{t} u_{\xi} D_{t}^{\alpha} \xi, \\
& D_{x}^{\alpha} u=\sigma_{x} u_{\xi} D_{x}^{\alpha} \xi
\end{aligned}
$$

where $\sigma_{t}$ and $\sigma_{x}$ are the fractal indexes $[43,48]$; without loss of generality, take $\sigma_{t}=\sigma_{x}=\kappa$, where $\kappa$ is a constant.

Step 2. Suppose that the solution of ODE (47) can be expressed by a polynomial in $\exp (-\theta(\xi))$ as follows:

$$
\begin{gathered}
u(\xi)=S_{1}(\theta)=\frac{\sum_{k=0}^{N_{1}} A_{k}[\exp (-\theta(\xi))]^{k}}{\sum_{k=0}^{M_{1}} B_{k}[\exp (-\theta(\xi))]^{k}}, \\
v(\xi)=S_{2}(\theta)=\frac{\sum_{k=0}^{N_{2}} C_{k}[\exp (-\theta(\xi))]^{k}}{\sum_{k=0}^{M_{2}} D_{k}[\exp (-\theta(\xi))]^{k}},
\end{gathered}
$$

where $A_{k}(0 \leq k \leq m)$ and $B_{k}(1 \leq k \leq n)$ are constants to be found such that $A_{N_{1}} \neq 0, C_{N_{2}} \neq 0$, and $\theta=\theta(\xi)$ satisfy the below ordinary differential equation:

$$
\theta^{\prime}=\mu \exp (\theta)+\exp (-\theta)+\lambda
$$

the solutions of equation (51) is given by

Family 1: if $\mu \neq 0$ and $\lambda^{2}-4 \mu>0$, afterwards $\theta(\xi)=$ $\ln \left[(-\lambda / 2 \mu)-\left(\sqrt{\lambda^{2}-4 \mu} / 2 \mu\right) \tanh \left(\left(\sqrt{\lambda^{2}-4 \mu} / 2\right) \bar{\xi}\right)\right]$

Family 2: if $\mu \neq 0$ and $\lambda^{2}-4 \mu<0$, afterwards $\theta(\xi)=$ $\ln \left[(-\lambda / 2 \mu)+\left(\sqrt{4 \mu-\lambda^{2}} / 2 \mu\right) \tanh \left(\left(\sqrt{4 \mu-\lambda^{2}} / 2\right) \bar{\xi}\right)\right]$

Family 3: if $\mu=0, \lambda \neq 0$ and $\lambda^{2}-4 \mu>0$, afterwards $\theta(\xi)=-\ln [\lambda / \exp (\lambda \bar{\xi})-1]$

Family 4: if $\mu \neq 0, \lambda \neq 0$ and $\lambda^{2}-4 \mu=0$, afterwards $\theta(\xi)=\ln \left[-\left(2 \lambda \bar{\xi}+4 / \lambda^{2} \bar{\xi}\right)\right]$

Family 5: if $\mu=0, \lambda=0$ and $\lambda^{2}-4 \mu=0$, afterwards $\theta(\xi)=\ln [\bar{\xi}] \quad$ where $A_{k}\left(k=0,1, \ldots, N_{1}\right), \quad B_{k}(k=$ $\left.0,1, \ldots, M_{1}\right), \quad C_{k}\left(k=0,1, \ldots, N_{2}\right), D_{k}(k=1,2, \ldots$, $\left.M_{2}\right), \lambda$, and $\mu$ are also the free constants.

Step 3. To determine the positive integer $N_{1}, M_{1}, N_{2}, M_{2}$, it, usually, can be accomplished by the nonlinear terms of highest-order in equation (47) with the highest-order linear terms. Substituting equations (50) and (51) into equation (13) yields an equation of powers of $\exp (-k \theta)$.

Step 4. With $N_{1}, M_{1}, N_{2}, M_{2}$ determined, we then collect all coefficients of powers of $\exp (-k \theta)$ in the resulting equation where these coefficients have to vanish. This will give a system of algebraic equations involving the parameters $A_{k}\left(k=0,1, \ldots, N_{1}\right), B_{k}(k=$ $\left.0,1, \ldots, M_{1}\right), C_{k}\left(k=0,1, \ldots, N_{2}\right), D_{k}$ $\left(k=1,2, \ldots, M_{2}\right), \lambda$, and $\mu$.

4.1. Periodic, Soliton, Singular, and Kink-Singular Solutions of the FCJM Equation by MEM. Balancing $u$ '"' with $v^{2} u^{\prime}$ and $v^{\prime \prime}$ with $v^{2} v^{\prime}$ in equation (19) gives the following cases:

$$
\begin{aligned}
& \text { Case I: }\left\{\begin{array}{l}
N_{1}=2, \\
M_{1}=0, \\
N_{2}=1, \\
M_{1}=0,
\end{array}\right. \\
& \text { Case II: }\left\{\begin{array}{l}
N_{1}=3, \\
M_{1}=1, \\
N_{2}=2, \\
M_{1}=1 .
\end{array}\right.
\end{aligned}
$$

Putting the values of (52) and $B_{0}=D_{0}=1$ to search for different wave solutions of the fractional CJM equations, we would like to start from ansatz as the following form:

$$
\begin{aligned}
& u(\xi)=A_{0}+A_{1} \exp (-\theta(\xi))+A_{2} \exp (-2 \theta(\xi)), \\
& v(\xi)=C_{0}+C_{1} \exp (-\theta(\xi)),
\end{aligned}
$$

where $A_{0}, A_{1}, A_{2}, C_{0}$, and $C_{1}$ are unknown values. Inserting (53) into equation (56) and then collecting the coefficients in the front of the various functions containing $\exp (-\theta(\xi))$ are given as follows: 


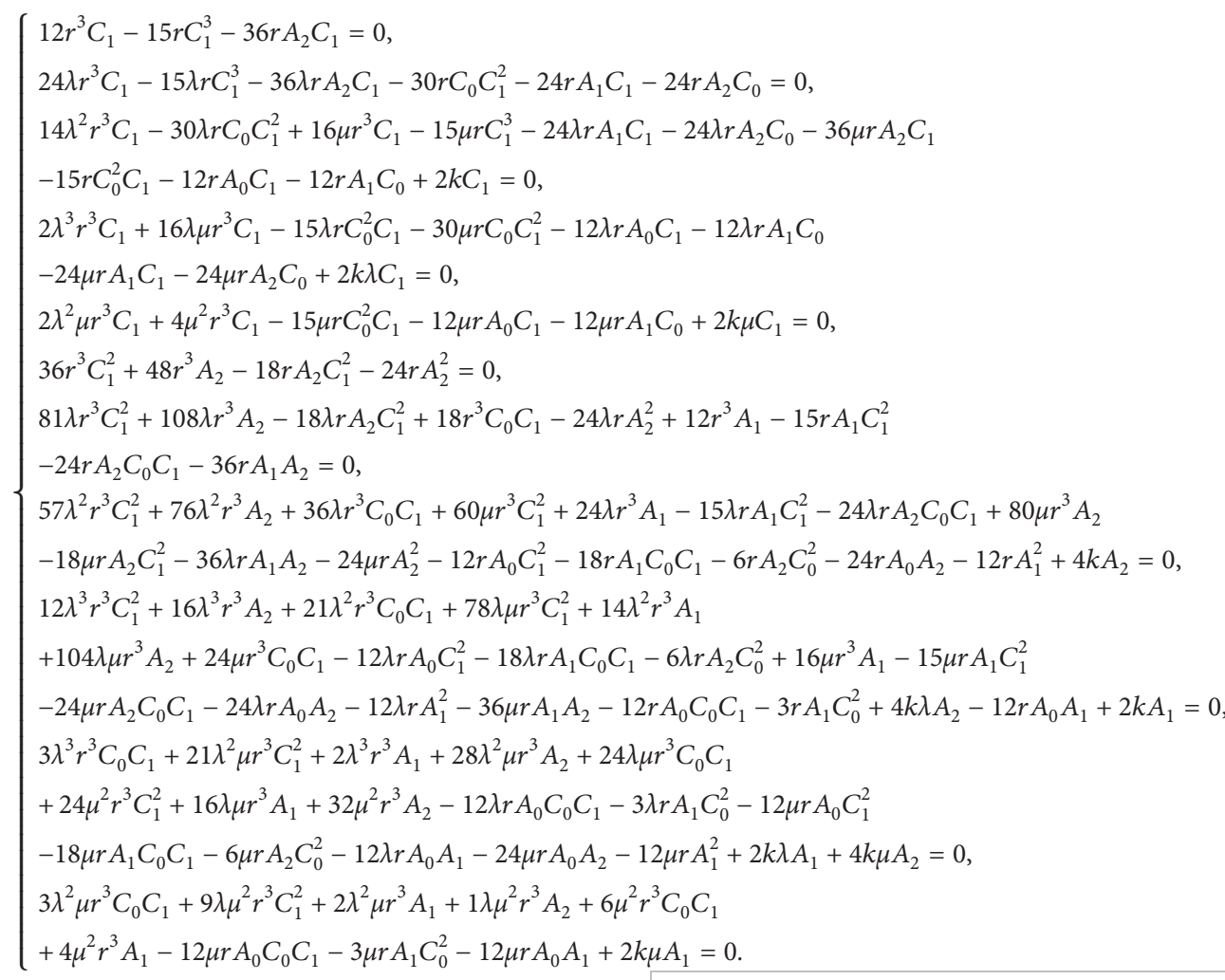

Solving the nonlinear algebraic system of equations can be concluded as the following solutions:

Set I:

$$
\left\{\begin{array}{l}
k=3 r C_{0}^{2}, \\
\lambda=0, \\
\mu=0, \\
r=r, \\
A_{0}=-\frac{1}{4} C_{0}^{2}, \\
A_{1}=-\frac{1}{2} C_{0} r \sqrt{-1}, \\
A_{2}=\frac{3}{4} r^{2}, \\
C_{0}=C_{0}, \\
C_{1}=r \sqrt{-1} .
\end{array}\right.
$$

$$
\begin{aligned}
& u_{1}=-\frac{1}{4} C_{0}^{2}-\frac{1}{2} C_{0} r \sqrt{-1} \frac{1}{\bar{\xi}}+\frac{3}{4} r^{2} \frac{1}{\bar{\xi}^{2}} \\
& v_{1}=C_{0}+r \sqrt{-1} \frac{1}{\bar{\xi}} \\
& \bar{\xi}=r x+3 r C_{0}^{2} \frac{t^{\alpha}}{\Gamma(\alpha+1)}+C .
\end{aligned}
$$

Set II:

$$
\begin{aligned}
& k=-\lambda^{2} r^{3}, \\
& \lambda=\lambda, \\
& \mu=0, \\
& r=r, \\
& A_{0}=0, \\
& A_{1}=\frac{1}{2} \lambda r^{2}, \\
& A_{2}=\frac{3}{4} r^{2}, \\
& C_{0}=0, \\
& C_{1}=r \sqrt{-1} .
\end{aligned}
$$

Plugging (55) into (53), we perceive a periodic solution of equation (18) by utilizing Family 1 as follows: 
Appending (57) into (20), we gain a hyperbolic solution of equation (18) by utilizing Family 3 as follows:

$$
\begin{aligned}
& u_{2}=\frac{1}{2} \lambda r^{2}\left[\frac{\exp (\lambda \bar{\xi})-1}{\lambda}\right]+\frac{3}{4} r^{2}\left[\frac{\exp (\lambda \bar{\xi})-1}{\lambda}\right]^{2}, \\
& v_{2}=r \sqrt{-1}\left[\frac{\exp (\lambda \bar{\xi})-1}{\lambda}\right], \\
& \bar{\xi}=r x-\lambda^{2} r^{3} \frac{t^{\alpha}}{\Gamma(\alpha+1)}+C .
\end{aligned}
$$

Set III:

$$
\left\{\begin{array}{l}
k=-\frac{49}{4} \lambda^{2} r^{3}, \\
\lambda=\lambda \\
\mu=0 \\
r=r \\
A_{0}=\frac{9}{16} \lambda^{2} r^{2}, \\
A_{1}=-\frac{1}{4} \lambda r^{2} \\
C_{1}=r \sqrt{-1} \\
A_{0}=-\frac{3}{2} \lambda r \sqrt{-1} r^{2} \\
A_{2}
\end{array}\right.
$$

Appending (59) into (20), we perceive a hyperbolic solution of equation (18) by utilizing Family 3 as follows:

$$
\begin{aligned}
& u_{3}=\frac{9}{16} \lambda^{2} r^{2}-\frac{1}{4} \lambda r^{2}\left[\frac{\exp (\lambda \bar{\xi})-1}{\lambda}\right]+\frac{3}{4} r^{2}\left[\frac{\exp (\lambda \bar{\xi})-1}{\lambda}\right]^{2}, \\
& v_{3}=-\frac{3}{2} \lambda r \sqrt{-1}+r \sqrt{-1}\left[\frac{\exp (\lambda \bar{\xi})-1}{\lambda}\right], \\
& \bar{\xi}=r x-\frac{49}{4} \lambda^{2} r^{3} \frac{t^{\alpha}}{\Gamma(\alpha+1)}+C .
\end{aligned}
$$

Set IV:

$$
\left\{\begin{array}{l}
k=r C_{0}^{2}, \\
\lambda=-\frac{C_{0} \sqrt{-1}}{r}, \\
\mu=0, \\
r=r, \\
A_{0}=-\frac{1}{4} C_{0}^{2}, \\
A_{1}=-2 C_{0} r \sqrt{-1}, \\
C_{1}=2 r \sqrt{-1} . \\
C_{0}=C_{0},
\end{array}\right.
$$

Appending (61) into (20), we perceive a hyperbolic solution of equation (18) by utilizing Family 3 as follows:

$$
\begin{aligned}
& u_{4}=-\frac{1}{4} C_{0}^{2}-2 C_{0} r \sqrt{-1}\left[\frac{\exp (\lambda \bar{\xi})-1}{\lambda}\right]+2 r^{2}\left[\frac{\exp (\lambda \bar{\xi})-1}{\lambda}\right]^{2}, \\
& v_{4}=C_{0}+2 r \sqrt{-1}\left[\frac{\exp (\lambda \bar{\xi})-1}{\lambda}\right], \\
& \bar{\xi}=r x+r C_{0}^{2} \frac{t^{\alpha}}{\Gamma(\alpha+1)}+C .
\end{aligned}
$$


Set V:

$$
\begin{aligned}
& k=\frac{21}{16} r C_{0}^{2}, \\
& \lambda=-\frac{3}{4} \frac{C_{0} \sqrt{-1}}{r}, \\
& \mu=0, \\
& r=r, \\
& A_{0}=-\frac{1}{4} C_{0}^{2}, \\
& A_{1}=-\frac{7}{8} C_{0} r \sqrt{-1}, \\
& C_{0}=C_{0}, \\
& A_{2}=\frac{3}{4} r^{2},
\end{aligned}
$$

Appending (63) into (20), we receive a hyperbolic solution of equation (18) by utilizing Family 3 as follows:

$$
\begin{aligned}
& u_{5}=-\frac{1}{4} C_{0}^{2}-\frac{7}{8} C_{0} r \sqrt{-1}\left[\frac{\exp (\lambda \bar{\xi})-1}{\lambda}\right]+\frac{3}{4} r^{2}\left[\frac{\exp (\lambda \bar{\xi})-1}{\lambda}\right]^{2}, \\
& v_{5}=C_{0}+2 r \sqrt{-1}\left[\frac{\exp (\lambda \bar{\xi})-1}{\lambda}\right], \\
& \bar{\xi}=r x+\frac{21}{16} r C_{0}^{2} \frac{t^{\alpha}}{\Gamma(\alpha+1)}+C .
\end{aligned}
$$

\section{Set VI:}

$$
\left\{\begin{array}{l}
k=-r^{3}\left(\lambda^{2}-4 \mu\right), \\
\lambda=\lambda \\
\mu=\mu, \\
r=r \\
A_{0}=\frac{1}{4} r^{2}\left(\lambda^{2}+4 \mu\right), \\
A_{1}=2 \lambda r^{2} \\
A_{2}=2 r^{2} \\
C_{0}=\lambda r \sqrt{-1}
\end{array}\right.
$$

Appending (65) into (20), we gain a hyperbolic solution of equation (18) by utilizing Family 1 as follows:

$$
\begin{aligned}
u_{6}= & \frac{1}{4} r^{2}\left(\lambda^{2}+4 \mu\right)+2 \lambda r^{2}\left[\frac{-\lambda}{2 \mu}-\frac{\sqrt{\lambda^{2}-4 \mu}}{2 \mu} \tanh \left(\frac{\sqrt{\lambda^{2}-4 \mu}}{2} \bar{\xi}\right)\right]^{-1} \\
& +2 r^{2}\left[\frac{-\lambda}{2 \mu}-\frac{\sqrt{\lambda^{2}-4 \mu}}{2 \mu} \tanh \left(\frac{\sqrt{\lambda^{2}-4 \mu}}{2} \bar{\xi}\right)\right]^{-2}, \\
v_{6}= & \lambda r \sqrt{-1}+2 r \sqrt{-1}\left[\frac{-\lambda}{2 \mu}-\frac{\sqrt{\lambda^{2}-4 \mu}}{2 \mu} \tanh \left(\frac{\sqrt{\lambda^{2}-4 \mu}}{2} \bar{\xi}\right)\right]^{-1}, \\
\bar{\xi}= & r x-r^{3}\left(\lambda^{2}-4 \mu\right) \frac{t^{\alpha}}{\Gamma(\alpha+1)}+C,
\end{aligned}
$$

where $\lambda^{2}-4 \mu>0$. Plugging (65) into (20), we perceive a hyperbolic solution of equation (18) by utilizing Family 2 as follows: 


$$
\begin{aligned}
u_{7}= & \frac{1}{4} r^{2}\left(\lambda^{2}+4 \mu\right)+2 \lambda r^{2}\left[\frac{-\lambda}{2 \mu}+\frac{\sqrt{4 \mu-\lambda^{2}}}{2 \mu} \tan \left(\frac{\sqrt{4 \mu-\lambda^{2}}}{2} \bar{\xi}\right)\right]^{-1} \\
& +2 r^{2}\left[\frac{-\lambda}{2 \mu}+\frac{\sqrt{4 \mu-\lambda^{2}}}{2 \mu} \tan \left(\frac{\sqrt{4 \mu-\lambda^{2}}}{2} \bar{\xi}\right)\right]^{-2}, \\
v_{7}= & \lambda r \sqrt{-1}+2 r \sqrt{-1}\left[\frac{-\lambda}{2 \mu}+\frac{\sqrt{4 \mu-\lambda^{2}}}{2 \mu} \tan \left(\frac{\sqrt{4 \mu-\lambda^{2}}}{2} \bar{\xi}\right)\right]^{-1}, \\
\bar{\xi}= & r x-r^{3}\left(\lambda^{2}-4 \mu\right) \frac{t^{\alpha}}{\Gamma(\alpha+1)}+C,
\end{aligned}
$$

where $\lambda^{2}-4 \mu<0$.

Set VII:

Appending (68) into (20), we get a hyperbolic solution of equation (18) by utilizing Family 1 as follows:

$$
\left\{\begin{array}{l}
k=\left(\lambda^{2} C_{1}^{2}-3 \lambda C_{0} C_{1}-\mu C_{1}^{2}+3 C_{0}^{2}\right) C_{1} \sqrt{-1}, \\
\lambda=\lambda \\
\mu=\mu \\
r=C_{1} \sqrt{-1} \\
A_{0}=-\frac{1}{2} \mu C_{1}^{2}-\frac{1}{4} C_{0}^{2} \\
A_{1}=-\frac{1}{2} C_{1}\left(\lambda C_{1}+C_{0}\right) \\
A_{2}=-\frac{3}{4} C_{1}^{2} \\
C_{0}=C_{0} \\
C_{1}=C_{1}
\end{array}\right.
$$

$$
\begin{aligned}
u_{8}= & -\frac{1}{2} \mu C_{1}^{2}-\frac{1}{4} C_{0}^{2}+-\frac{1}{2} C_{1}\left(\lambda C_{1}+C_{0}\right)\left[\frac{-\lambda}{2 \mu}-\frac{\sqrt{\lambda^{2}-4 \mu}}{2 \mu} \tanh \left(\frac{\sqrt{\lambda^{2}-4 \mu}}{2} \bar{\xi}\right)\right]^{-1} \\
& -\frac{3}{4} C_{1}^{2}\left[\frac{-\lambda}{2 \mu}-\frac{\sqrt{\lambda^{2}-4 \mu}}{2 \mu} \tanh \left(\frac{\sqrt{\lambda^{2}-4 \mu}}{2} \bar{\xi}\right)\right]^{-2}, \\
v_{8}= & C_{0}+C_{1}\left[\frac{-\lambda}{2 \mu}-\frac{\sqrt{\lambda^{2}-4 \mu}}{2 \mu} \tanh \left(\frac{\sqrt{\lambda^{2}-4 \mu}}{2} \bar{\xi}\right)\right]^{-1}, \\
\bar{\xi}= & C_{1} \sqrt{-1} x+\left(\lambda^{2} C_{1}^{2}-3 \lambda C_{0} C_{1}-\mu C_{1}^{2}+3 C_{0}^{2}\right) C_{1} \sqrt{-1} \frac{t^{\alpha}}{\Gamma(\alpha+1)}+C
\end{aligned}
$$




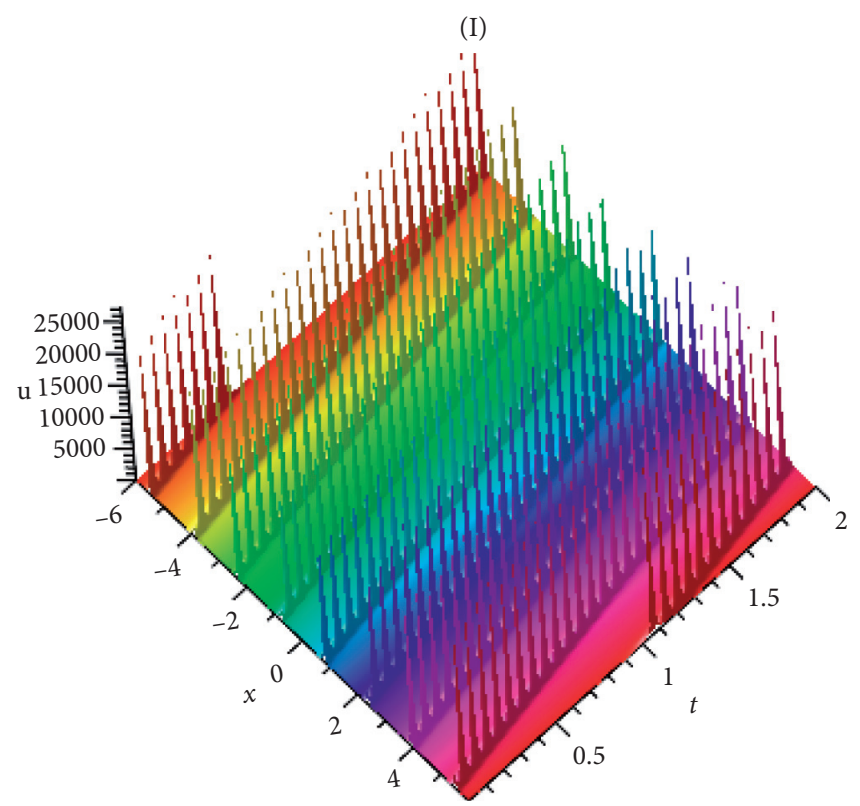

(II)

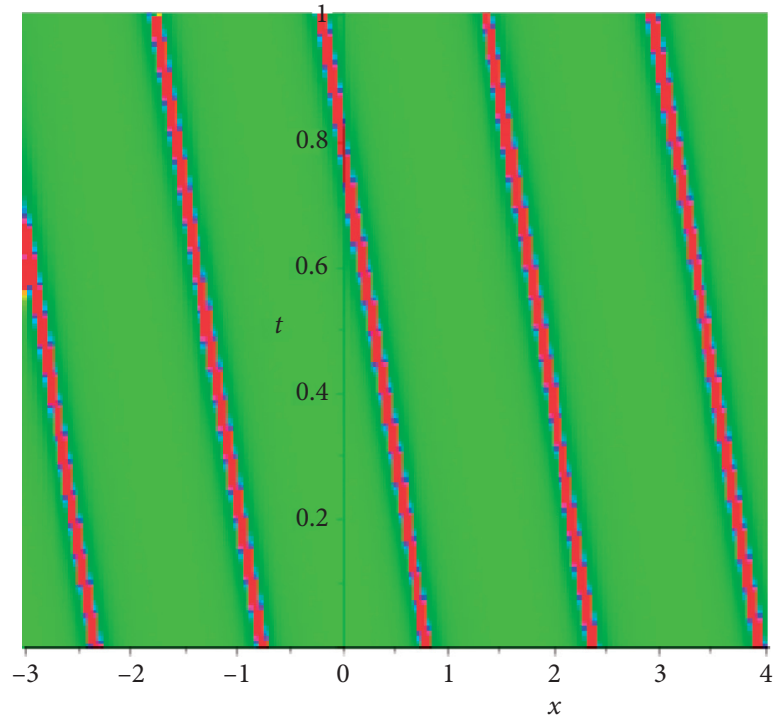

(III)
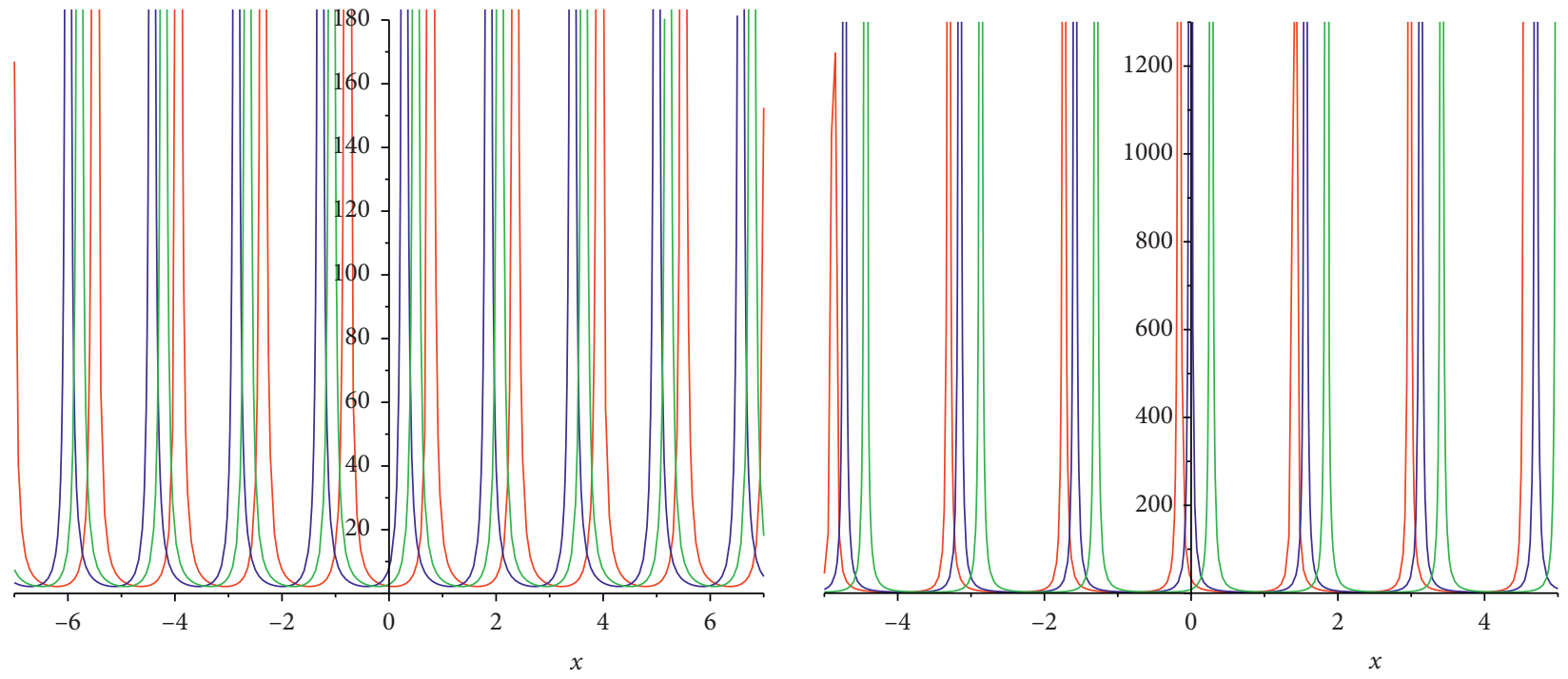

$t=0$
$t=0.5$
$t=5$

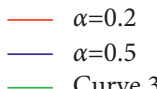

FIGURE 1: Evolution of the periodic solution (23) with data $r=, k_{1}=1, k_{2}=2, k_{3}=3, \alpha=1$. 
(I)

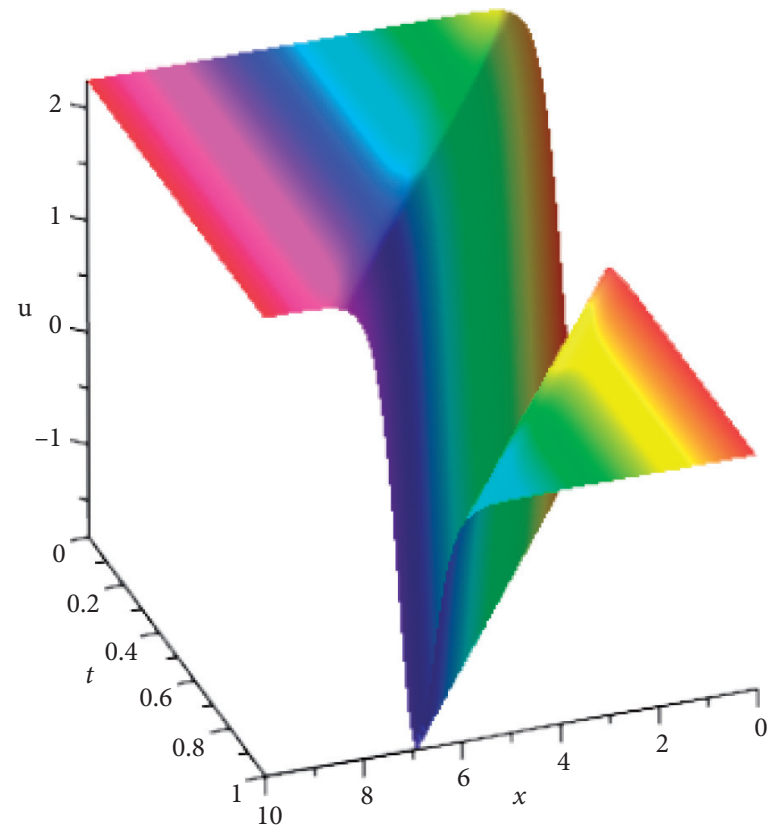

(III)

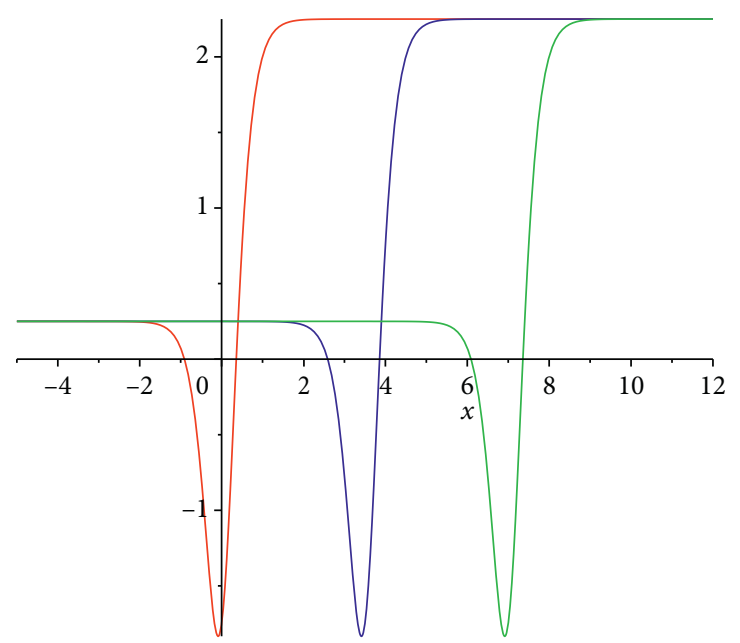

- $\mathrm{t}=0$

- $\mathrm{t}=0.5$

$\mathrm{t}=1$
(II)

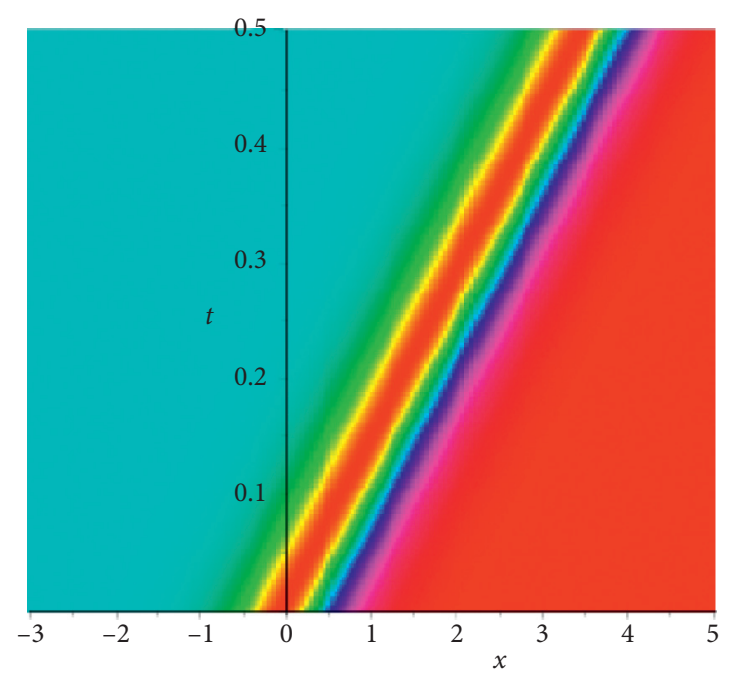

(IV)

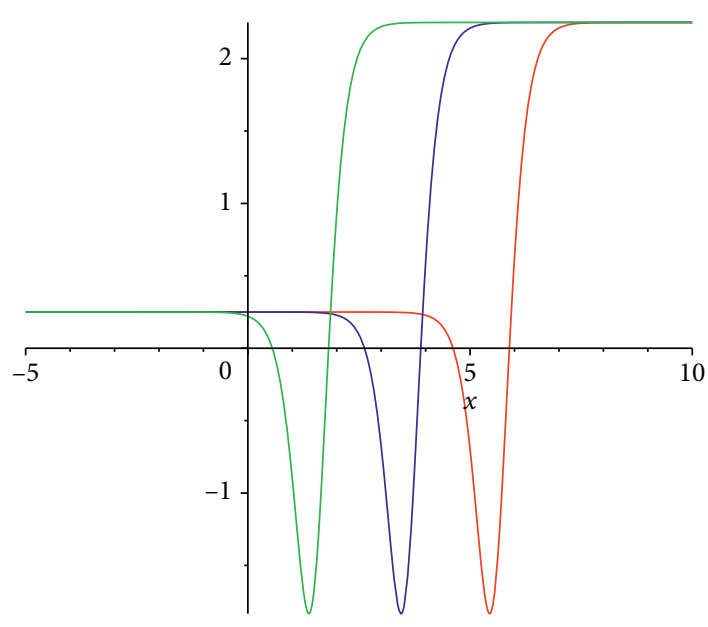

$$
\begin{aligned}
& \text { — } \alpha=0.2 \\
& \text { - } \alpha=0.5 \\
& \text { — } \alpha=0.98
\end{aligned}
$$

FIgURe 2: Evolution of the periodic solution (25) with data $r=, k_{1}=1, k_{2}=2, k_{3}=1, \alpha=1$. 
(I)

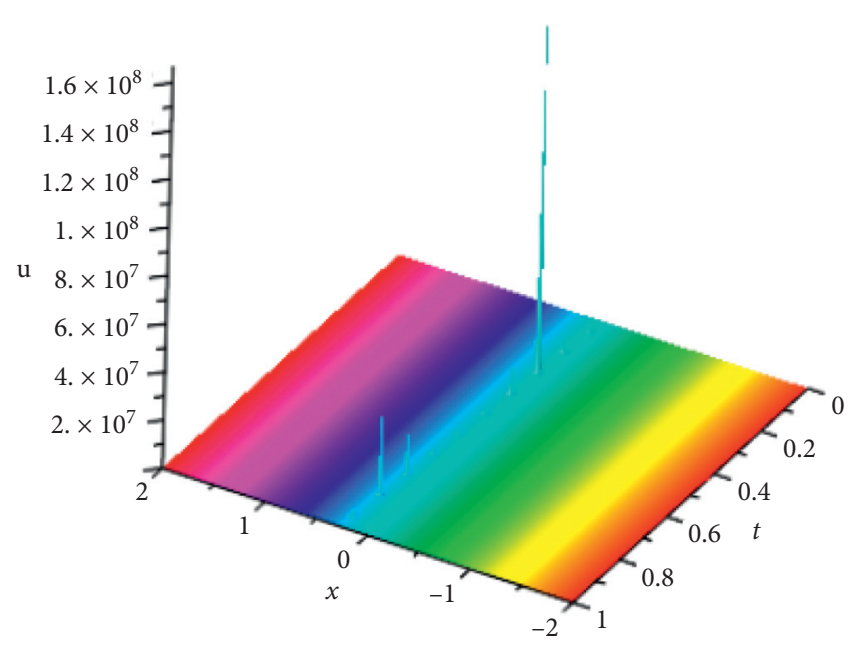

(III)

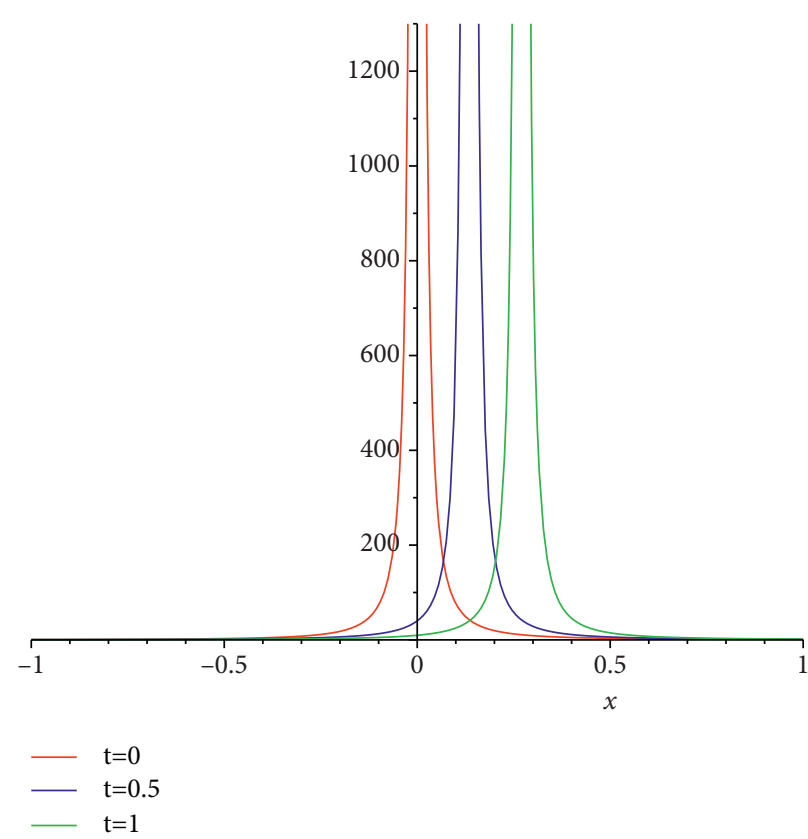

(II)

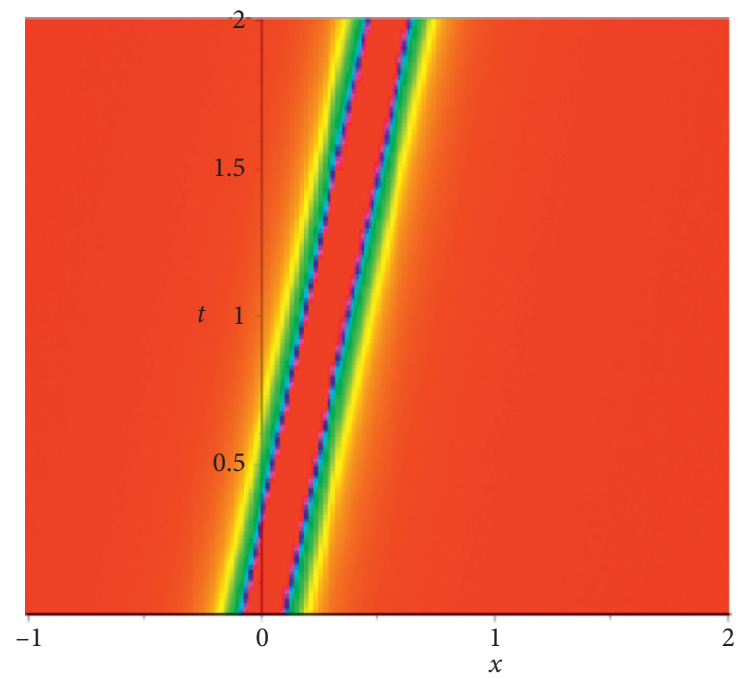

(IV)

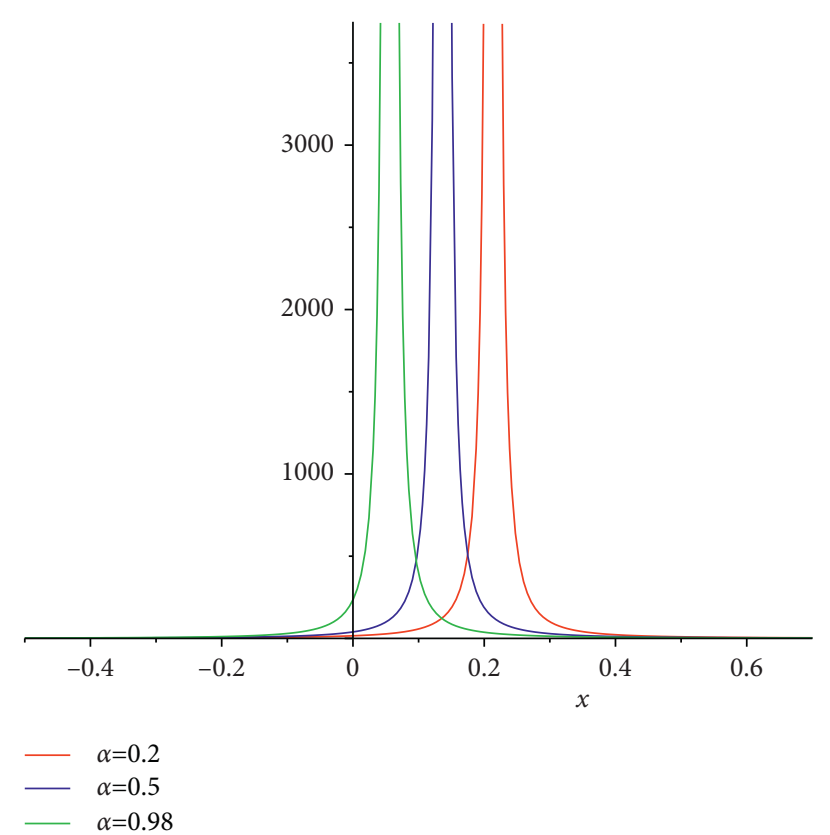

Figure 3: Evolution of the periodic solution (27) with data $r=2, k_{1}=0.3, k_{2}=0.4, k_{3}=0.5, \alpha=1$. 
(I)

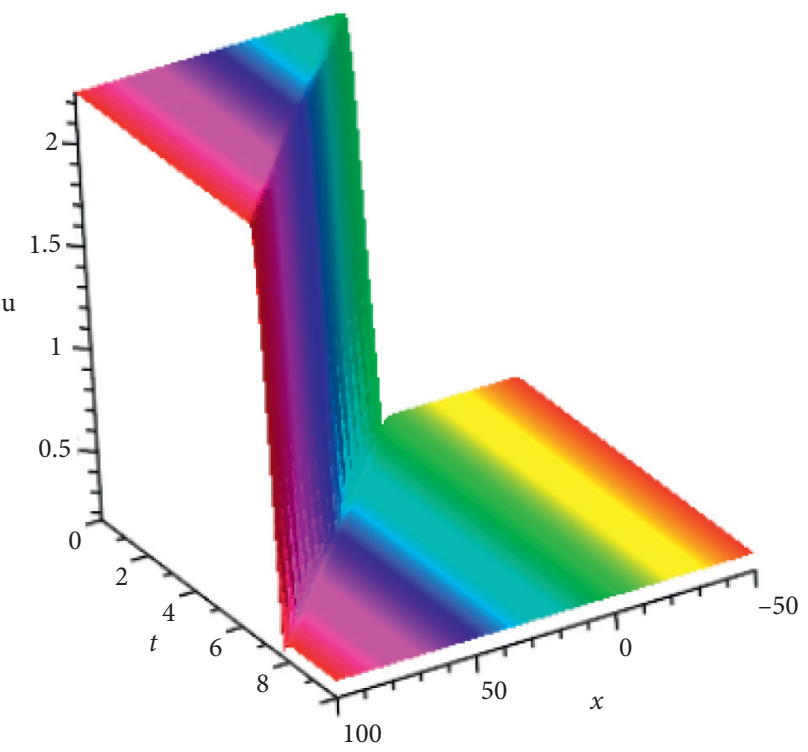

(III)

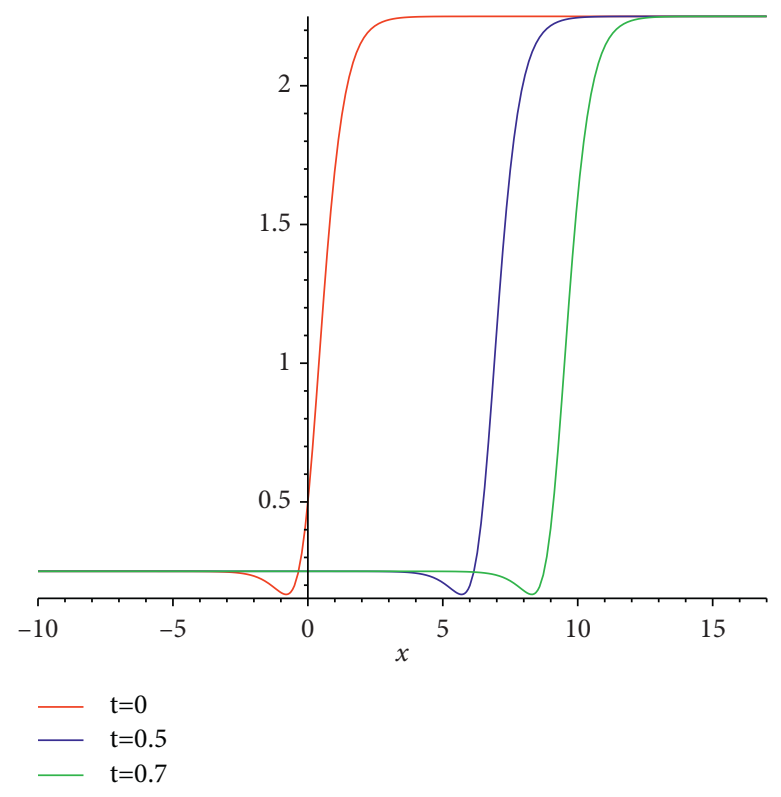

(II)

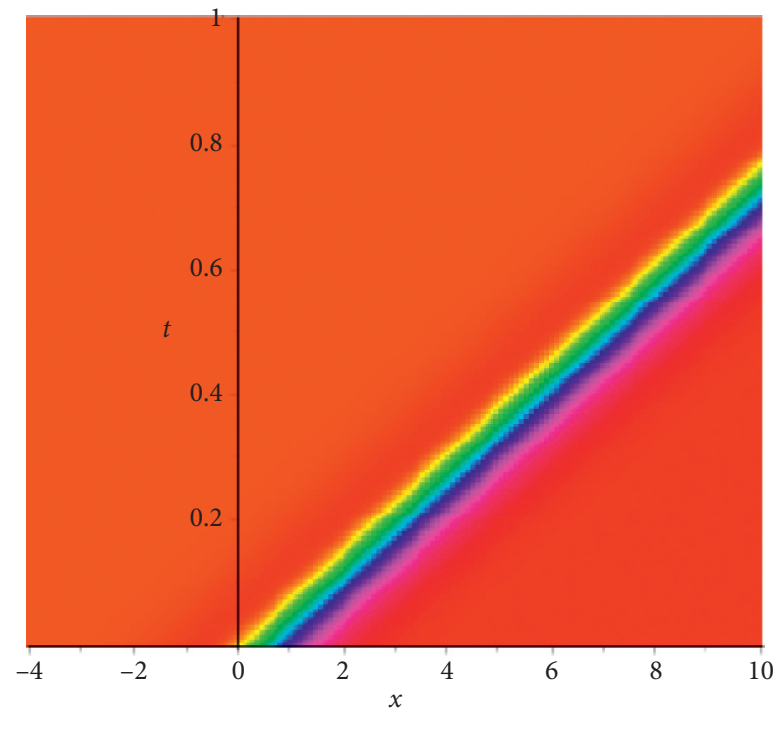

(IV)

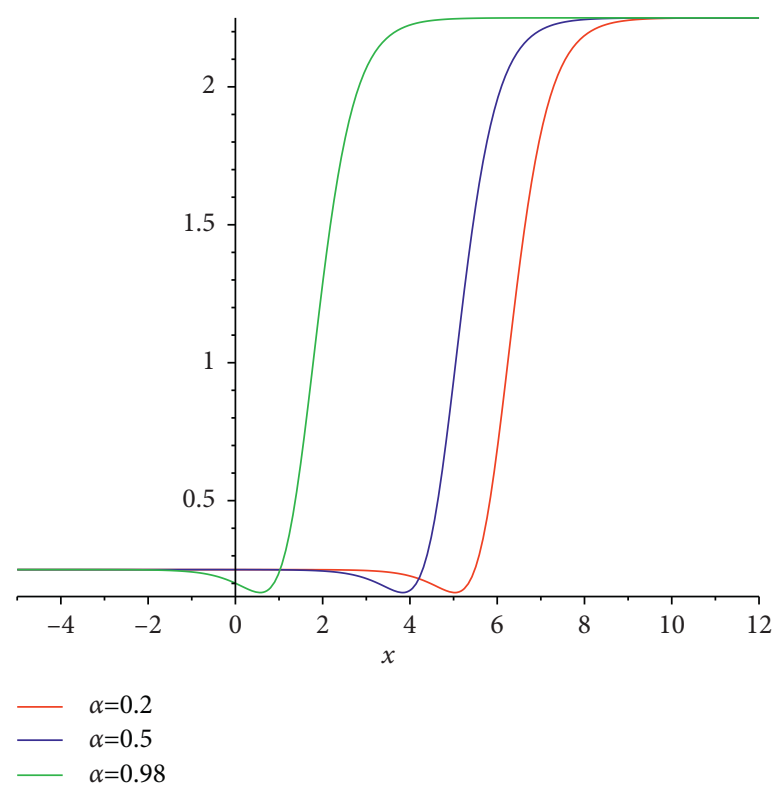

FIgURE 4: Evolution of the periodic solution (29) with data $r=2, k_{2}=1, k_{3}=2, \alpha=1$. 
(I)

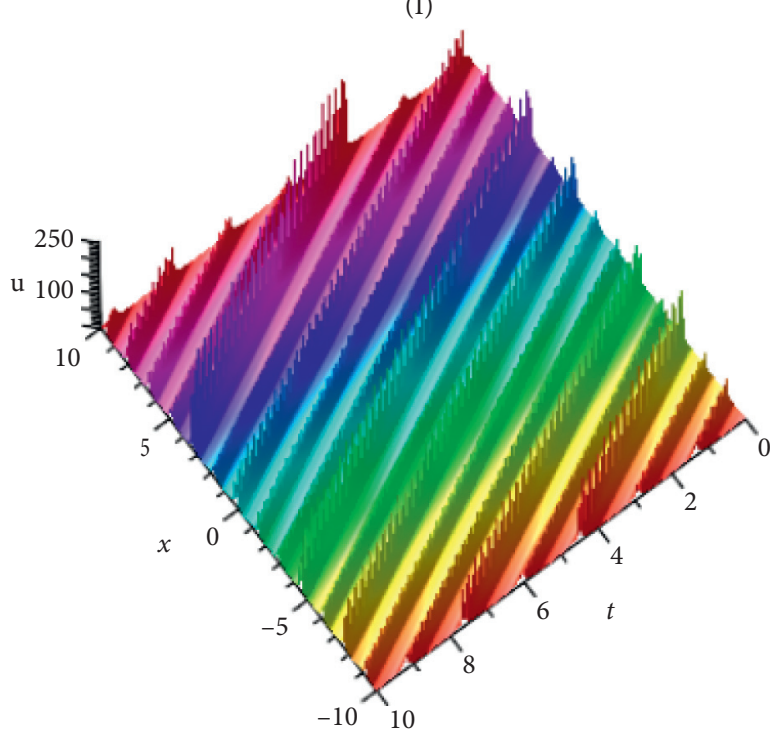

(III)

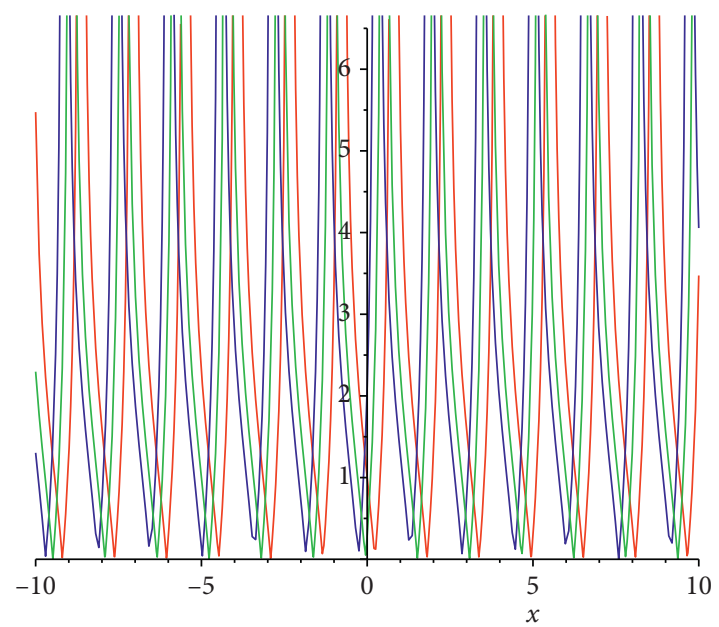

- $\mathrm{t}=0$
(II)

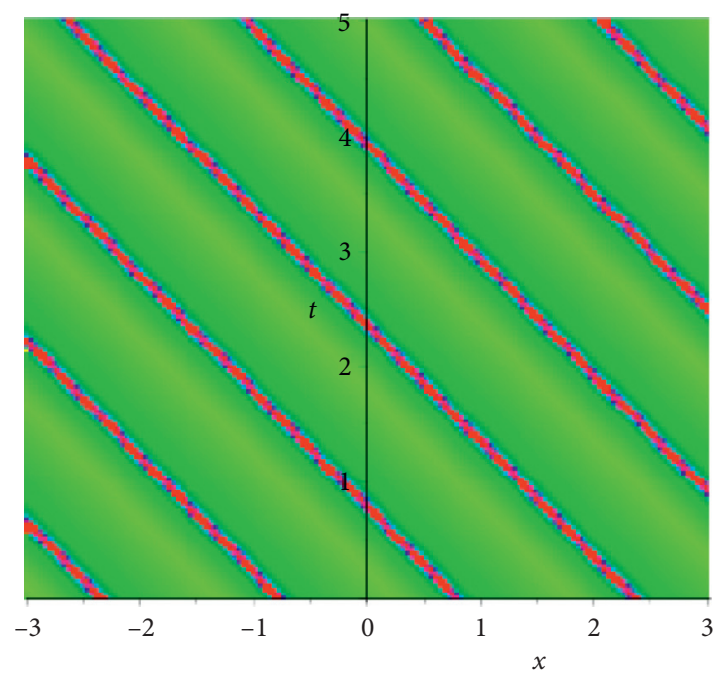

(IV)

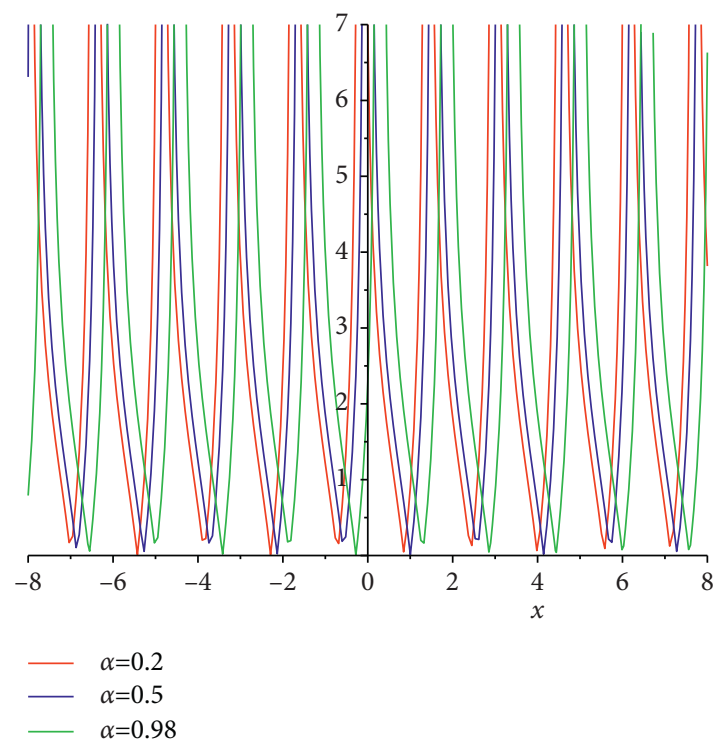

FIGURE 5: Evolution of the periodic solution (24) with data $r=, k_{1}=1, k_{2}=2, k_{3}=3, \alpha=1$. 
(I)

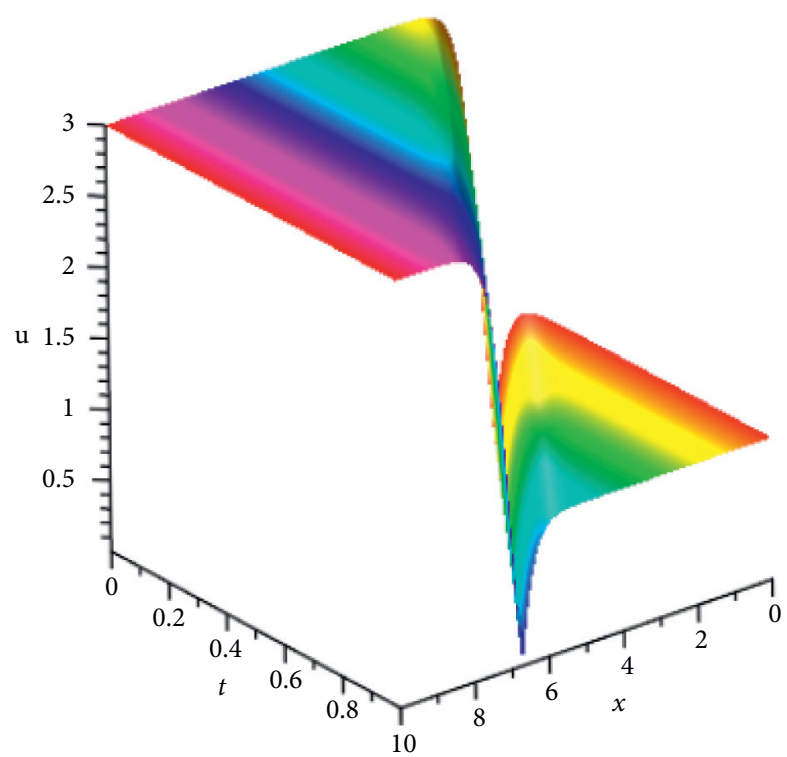

(III)

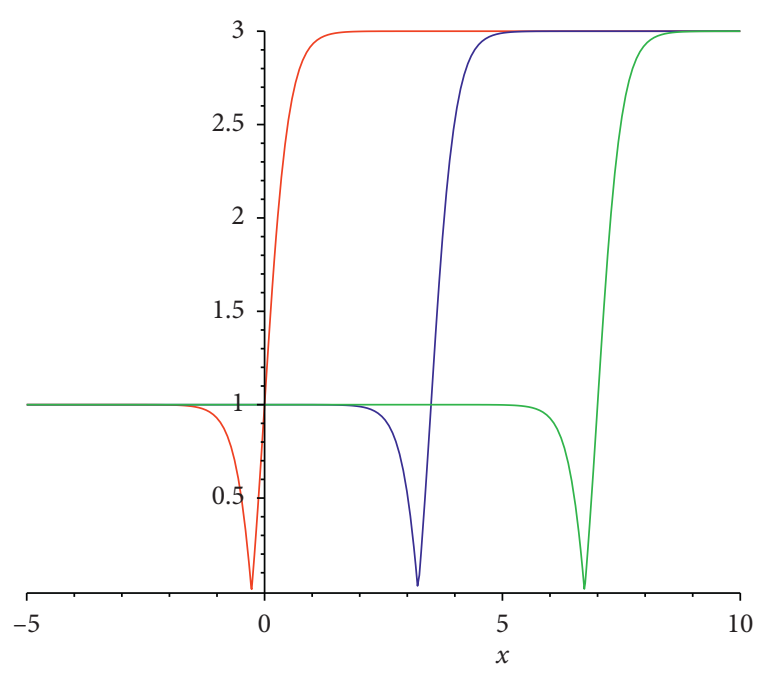

$-\begin{aligned} & \mathrm{t}=0 \\ & \mathrm{t}=0.5\end{aligned}$

$\mathrm{t}=1$
(II)

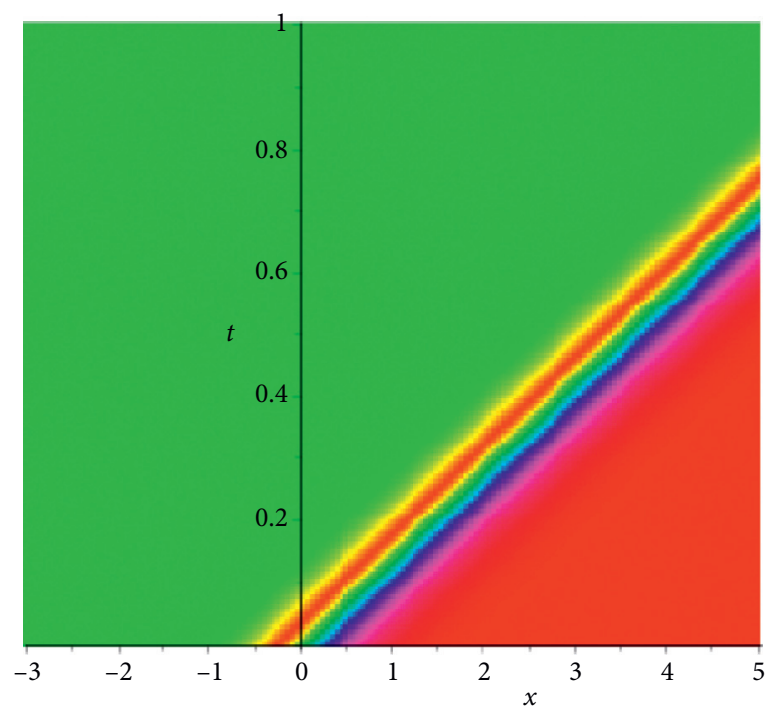

(IV)

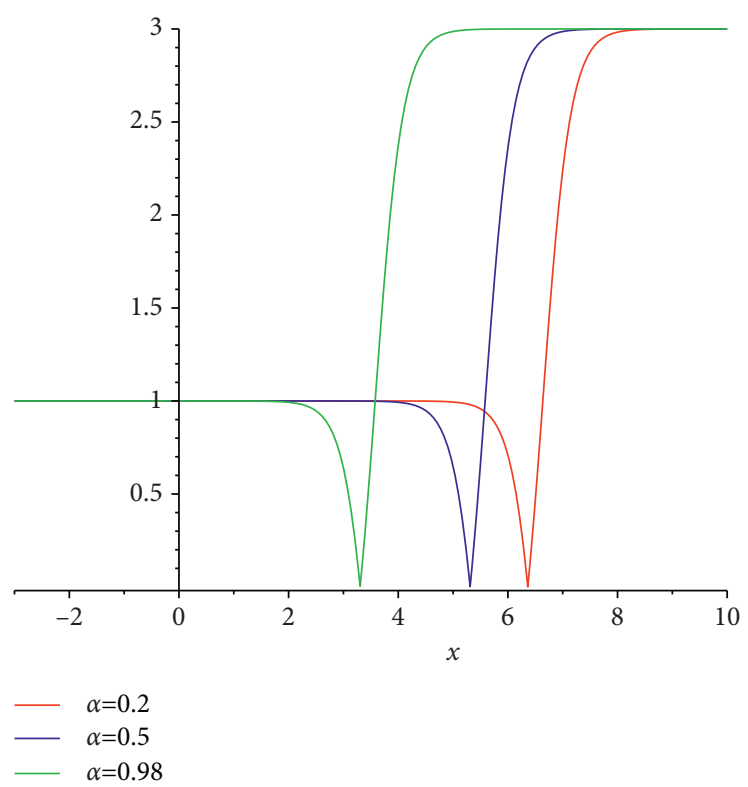

FIgURE 6: Evolution of the periodic solution (26) with data $r=, k_{1}=1, k_{2}=2, k_{3}=1, \alpha=1$. 
(I)

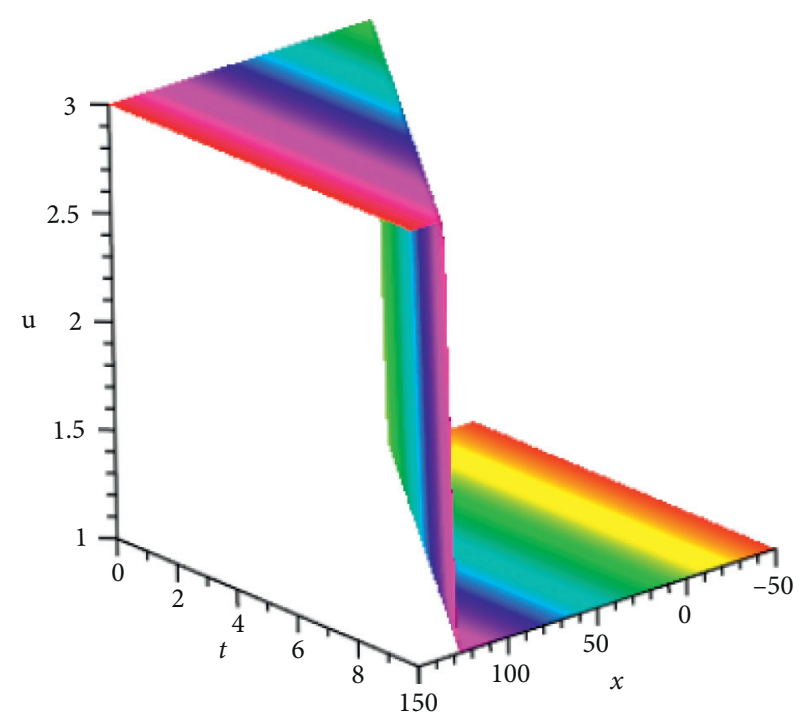

(III)

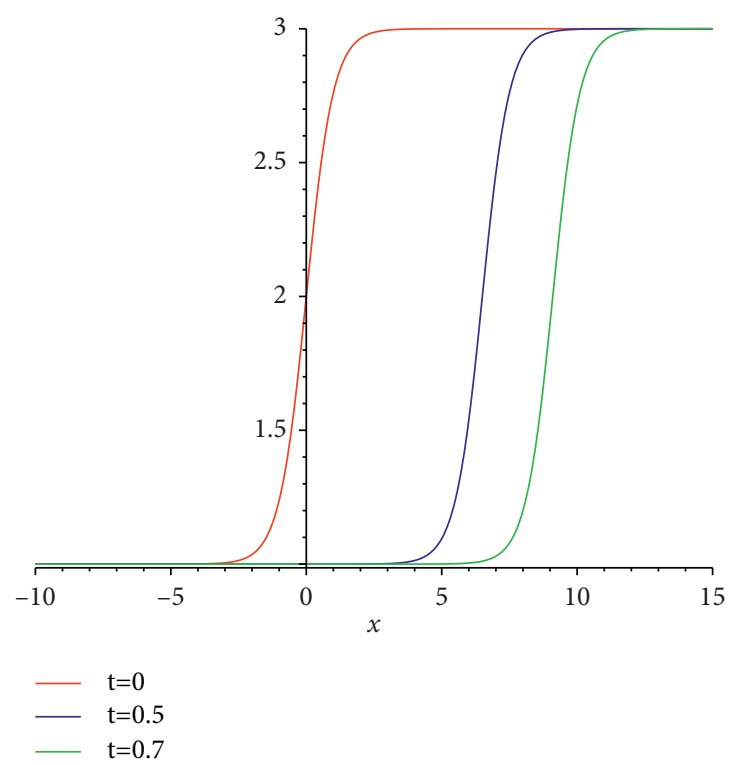

(II)

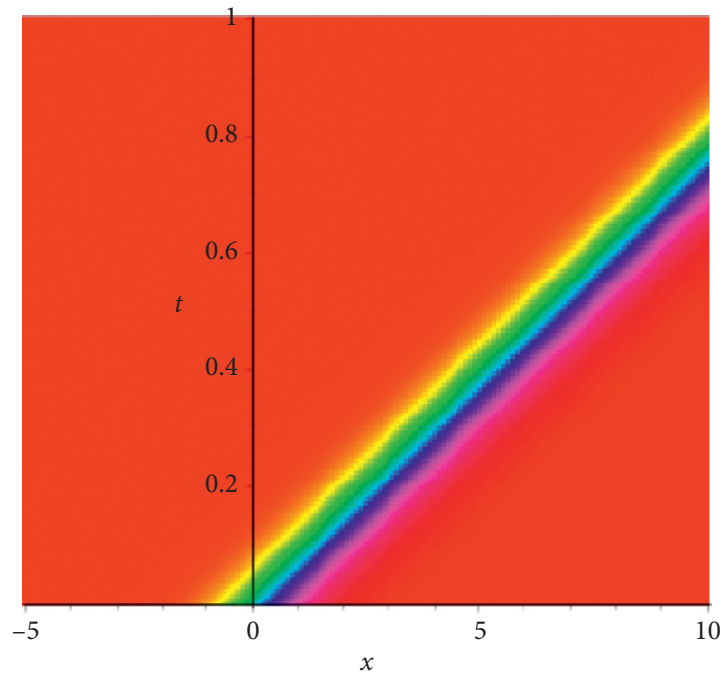

(IV)

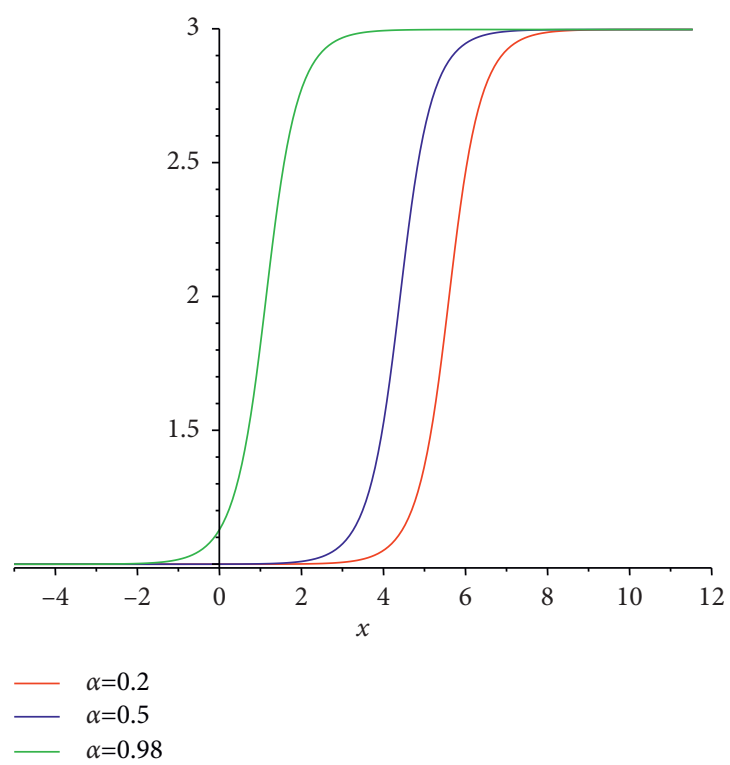

Figure 7: Evolution of the periodic solution (30) with data $r=, k_{1}=1, k_{2}=2, k_{3}=1, \alpha=1$. 
where $\lambda^{2}-4 \mu>0$. Appending (68) into (20), we perceive a hyperbolic solution of equation (18) by utilizing Family 2 as follows:

$$
\begin{aligned}
u_{9}= & -\frac{1}{2} \mu C_{1}^{2}-\frac{1}{4} C_{0}^{2}+-\frac{1}{2} C_{1}\left(\lambda C_{1}+C_{0}\right)\left[\frac{-\lambda}{2 \mu}+\frac{\sqrt{4 \mu-\lambda^{2}}}{2 \mu} \tan \left(\frac{\sqrt{4 \mu-\lambda^{2}}}{2} \bar{\xi}\right)\right]^{-1} \\
& -\frac{3}{4} C_{1}^{2}\left[\frac{-\lambda}{2 \mu}+\frac{\sqrt{4 \mu-\lambda^{2}}}{2 \mu} \tan \left(\frac{\sqrt{4 \mu-\lambda^{2}}}{2} \bar{\xi}\right)\right]^{-2}, \\
v_{9}= & C_{0}+C_{1}\left[\frac{-\lambda}{2 \mu}+\frac{\sqrt{4 \mu-\lambda^{2}}}{2 \mu} \tan \left(\frac{\sqrt{4 \mu-\lambda^{2}}}{2} \bar{\xi}\right)\right]^{-1}, \\
\bar{\xi}= & C_{1} \sqrt{-1} x+\left(\lambda^{2} C_{1}^{2}-3 \lambda C_{0} C_{1}-\mu C_{1}^{2}+3 C_{0}^{2}\right) C_{1} \sqrt{-1} \frac{t^{\alpha}}{\Gamma(\alpha+1)}+C,
\end{aligned}
$$

where $\lambda^{2}-4 \mu>0$.

\section{Interpretation Solutions with Physical Concepts}

This section shows some soliton solutions of fractional form of the coupled Jaulent-Miodek equation.

Remark 3. The three-dimensional dynamic plots of the wave and corresponding density designs, contour designs, and two-dimensional designs were successfully plotted in Figures $1-7$ by utilizing the Maple.

In order to analyze the dynamics characteristics briefly, we would like to discuss the evolution characteristic. By taking appropriate parameters of these parameters in the following, we set

$$
\left\{\begin{array}{l}
\text { Eq. (23): } r=2, k_{1}=1, k_{2}=2, k_{3}=3, \alpha=1 \\
\text { Eq. (25): } r=2, k_{1}=1, k_{2}=2, k_{3}=1, \alpha=1 \\
\text { Eq. (27): } r=2, k_{1}=0.3, k_{2}=0.4, k_{3}=0.5, \alpha=1, \\
\text { Eq. (29): } r=2, k_{2}=1, k_{3}=2, \alpha=1 \\
\text { Eq. (24): } r=2, k_{1}=1, k_{2}=2, k_{3}=3, \alpha=1 \\
\text { Eq. (26): } r=2, k_{1}=1, k_{2}=2, k_{3}=1, \alpha=1 \\
\text { Eq. (30): } r=2, k_{1}=1, k_{2}=2, k_{3}=1, \alpha=1
\end{array}\right.
$$

We can see that the trigonometric function is as periodic solution, hyperbolic function is as soliton wave solution, exponential function is as kink solution, rational exponential function is as singular-kink solution, and rational function is as singular solution.

\section{Conclusion}

We have investigated in detail the time-fractional coupled Jaulent-Miodek equation. By utilizing the $\tan (\theta / 2)$-expansion technique and modified $\exp (-\theta(\xi))$-expansion technique, we find bright, dark soliton, analytically periodic, singular, and kink-soliton solutions with the constraints under which these solutions are obtained. The $\tan (\theta / 2)$-expansion technique and modified $\exp (-\theta(\xi))$-expansion technique are helpful to obtain solutions in the form of hyperbolic and trigonometric forms which are exact and helpful in understanding the fractional forms of it. Finally, a transformation is used to draw soliton solutions of system (1) by the use of Maple software. So, this gives the efficient applications of aforementioned techniques for fractional PDEs.

By utilizing Maple, the evolution phenomenon of these waves is seen in Figures 1-7, respectively. Moreover, we can discover the physical structure and properties of the new periodic wave, soliton, singular-kink, and bell soliton solutions for the time FCJM equations. These new solutions can be utilized to perceive their physical morphology and density curves by computer computations, which will be extensively used in optics, DWDM systems, metasurfaces, and metamaterials, and so forth.

\section{Data Availability}

The datasets supporting the conclusions of this article are included in the article.

\section{Conflicts of Interest}

The authors declare that they have no conflicts of interest.

\section{References}

[1] J. Manafian and M. Lakestani, "Application of $\tan (\phi / 2)$-expansion method for solving the Biswas-Milovic equation for Kerr law nonlinearity," Optik, vol. 127, no. 4, pp. 2040-2054, 2016.

[2] Z. Pinar, H. Rezazadeh, and M. Eslami, "Generalized logistic equation method for Kerr law and dual power law 
Schrodinger equations," Optical and Quantum Electronics, vol. 52, no. 504, pp. 1-16, 2020.

[3] K. S. Nisar, O. A. Ilhan, J. Manafian, M. Shahriari, and D. Soybaş, "Analytical behavior of the fractional Bogoyavlenskii equations with conformable derivative using two distinct reliable methods," Results in Physics, vol. 22, p. 103975, 2021.

[4] K. Khan and M. A. Akbar, "The $\exp (-\Phi(\xi))$-expansion method for finding travelling wave solutions of VakhnenkoParkes equation," International Journal of Dynamical Systems and Differential Equations, vol. 5, no. 1, pp. 72-83, 2014.

[5] K. Ayub, M. Y. Khan, A. Rani, Q. M. Ul Hassan, B. Ahmed, and M. Shakeel, "Application of the $\operatorname{Exp}(-\varphi(\zeta))$-expansion method for solitary wave solutions," Arab Journal of Basic and Applied Sciences, vol. 26, no. 1, pp. 376-384, 2019.

[6] J. Manafian and R. F. Zinati, "Application of $\tan (\phi / 2)$-expansion method to solve some nonlinear fractional physical model," Proceedings of the National Academy of Sciences, India Section A, vol. 88, no. 3, pp. 1-20, 2018.

[7] J. Manafian and M. R. Foroutan, "Application of $\tan (\phi / 2)$-expansion method for the time-fractional Kuramoto-Sivashinsky equation," Optical and Quantum Electronics, vol. 49, p. 172, 2017.

[8] M. A. E. Abdelrahman, E. H. M. Zahran, and M. M. A. Khater, "The exp (-\&phi; (\&xi;))-expansion method and its application for solving nonlinear evolution equations," International Journal of Modern Nonlinear Theory and Application, vol. 04, no. 01, pp. 37-47, 2015.

[9] S. Saha Ray, "New exact solutions of fractional DaveyStewartson equation with power-law nonlinearity and new integrable Davey-Stewartson-type equation," The European Physical Journal Plus, vol. 131, no. 9, p. 327, 2016.

[10] Z. Hammouch, T. Mekkaoui, and P. Agarwal, "Optical solitons for the Calogero-Bogoyavlenskii-Schiff equation in $(2+1)$ dimensions with time-fractional conformable derivative," The European Physical Journal Plus, vol. 133, no. 7, p. 248, 2018.

[11] J. Manafian, O. A. Ilhan, S. Y. Mohyaldeen, S. M. Zeynalli, and G. Singh, "New strategic method for fractional mitigating internet bottleneck with quadraticcubic nonlinearity," The Mathematical Scientist, 2021.

[12] H. Rezazadeh, J. Manafian, F. S. Khodadad, and F. Nazari, "Traveling wave solutions for density-dependent conformable fractional diffusion-reaction equation by the first integral method and the improved $\tan ((1 / 2)(\varphi(\xi)))$-expansion method," Optical and Quantum Electronics, vol. 50, no. 3, p. 121, 2018.

[13] F. Mohammadizadeh, S. Rashidi, and S. R. Hejazi, "Spacetime fractional Klein-Gordon equation: symmetry analysis, conservation laws and numerical approximations," Mathematics and Computers in Simulation, vol. 188, pp. 476-497, 2021.

[14] Y. E. Aghdam, H. Mesgarani, G. M. Moremedi, and M. Khoshkhahtinat, "High-accuracy numerical scheme for solving the space-time fractional advection-diffusion equation with convergence analysis," Alexandria Engineering Journal, vol. 6. , 2021 In press.

[15] A. M. Wazwaz, "Multiple kink solutions and multiple singular kink solutions for $(2+1)$-dimensional nonlinear models generated by the Jaulent-Miodek hierarchy," Physics Letters A, vol. 373, Article ID 18441846, 2009.

[16] J. He and L. Zhang, "Generalized solitary solution and compacton-like solution of the Jaulent-Miodek equations using the Exp-function method," Physics Letters A, vol. 372, Article ID 10441047, 2008.

[17] Y. Zhang, X. Liu, and G. Wang, "Symmetry reductions and exact solutions of the $(2+1)$-dimensional Jaulent-Miodek equation," Applied Mathematics and Computation, vol. 219, Article ID 911916, 2012.

[18] J. Biazar and M. Eslami, "A reliable algorithm for solving nonlinear Jaulent-Miodek equation," Journal of King Saud University Science, vol. 23, Article ID 1336137, 2011.

[19] A. S. Rashed and M. M. Kassem, "Hidden symmetries and exact solutions of integro-differential JaulentMiodek evolution equation," Applied Mathematics and Computation, vol. 247, Article ID 11411155, 2014.

[20] W. M. Taha and M. S. M. Noorani, "Exact solutions of equation generated by the Jaulent-Miodek hierarchy by $\left(G^{\prime} /\right.$ G)-Expansion method," Mathematical Problems in Engineering, vol. 2013, pp. 1-7, 2013.

[21] H. Liu and F. Yan, "The bifurcation and exact travelling wave solutions for $(2+1)$-dimensional nonlinear models generated by the Jaulent-Miodek hierarchy," International Journal of Nonlinear Science, vol. 11, no. 2, pp. 200-205, 2011.

[22] R. Sadat and M. Kassem, "Explicit solutions for the $(2+1)$ dimensional JaulentMiodek equation using the integrating factors method in an unbounded domain," Mathematical and Computational Applications, vol. 23, pp. 1-9, 2018.

[23] D. Kaya and S. M. El-Sayed, "A numerical method for solving Jaulent-Miodek equation," Physics Letters A, vol. 318, no. 4-5, pp. 345-353, 2003.

[24] R. Zhou, "The finite-band solution of the Jaulent-Miodek equation," Journal of Mathematical Physics, vol. 38, no. 5, pp. 2535-2546, 1997.

[25] G. Xu, "N-Fold Darboux transformation of the JaulentMiodek equation," Applied Mathematics, vol. 5, no. 17, pp. 2657-2663, 2014.

[26] A. K. Gupta and S. S. Ray, "An investigation with Hermite Wavelets for accurate solution of Fractional Jaulent-Miodek equation associated with energy-dependent Schrödinger potential," Applied Mathematics and Computation, vol. 270, pp. 458-471, 2015.

[27] H. Wang and T.-C. Xia, "The fractional supertrace identity and its application to the super Jaulent-Miodek hierarchy," Communications in Nonlinear Science and Numerical Simulation, vol. 18, no. 10, pp. 2859-2867, 2013.

[28] M. Jaulent and I. Miodek, "Nonlinear evolution equations associated with 'enegry-dependent Schrödinger potentials'," Letters in Mathematical Physics, vol. 1, no. 3, pp. 243-250, 1976.

[29] S. Sahoo and S. Saha Ray, "New solitary wave solutions of time-fractional coupled Jaulent-Miodek equation by using two reliable methods," Nonlinear Dynamics, vol. 85, no. 2, pp. 1167-1176, 2016.

[30] A. K. Gupta and S. S. Ray, "An investigation with Hermite Wavelets for accurate solution of Fractional Jaulent-Miodek equation associated with energy-dependent Schrödinger potential," Applied Mathematics and Computation, vol. 270, no. 1, pp. 458-471, 2015.

[31] A. Majlesi, H. R. Ghehsareh, and A. Zaghian, "On the fractional Jaulent-Miodek equation associated with energy-dependent Schrödinger potential: lie symmetry reductions, explicit exact solutions and conservation laws," The European Physical Journal Plus, vol. 132, pp. 1-14, 2015.

[32] J. G. Liu, M. Eslami, H. Rezazadeh, and M. Mirzazadeh, "The dynamical behavior of mixed type lump solutions on the $(3+1)$-dimensional generalized Kadomtsev-Petviashvili- 
Boussinesq equation," International Journal of Nonlinear Sciences and Numerical Stimulation, vol. 21, no. 7-8, pp. 661-665, 2020.

[33] H. Ma, Q. Cheng, and A. Deng, "N-soliton solutions and localized wave interaction solutions of a $(3+1)$-dimensional potential-Yu-Toda-Sasa-Fukuyamaf equation," Modern Physics Letters B, vol. 35, no. 10, p. 2150277, 2021.

[34] W.-X. Ma, "Lump solutions to the kadomtsev-petviashvili equation," Physics Letters A, vol. 379, no. 36, pp. 1975-1978, 2015.

[35] H. M. Baskonus, H. Bulut, and F. B. M. Belgacem, "Analytical solutions for nonlinear long-short wave interaction systems with highly complex structure," Journal of Computational and Applied Mathematics, vol. 312, no. 1, pp. 257-266, 2017.

[36] A. Boulkhemair, A. Chakib, and A. Sadik, "Geometrical variations of a state-constrained functional on star-shaped domains," Advanced Mathematical Models \& Applications, vol. 6, no. 2, pp. 73-88, 2021.

[37] A. Nachaoui, M. Nachaoui, and B. Gasimov, "Parallel numerical computation of an analytical method for solving an inverse problem," Advanced Mathematical Models \& Applications, vol. 6, no. 2, pp. 162-173, 2021.

[38] G. Jumarie, "Modified Riemann-Liouville derivative and fractional Taylor series of nondifferentiable functions further results," Computers \& Mathematics with Applications, vol. 51, no. 9-10, pp. 1367-1376, 2006.

[39] K. B. Oldham and J. Spanier, The Fractional Calculus, Academic Press, New York, NY, USA, 1974.

[40] C.-s. Liu, "Counterexamples on Jumarie's two basic fractional calculus formulae," Communications in Nonlinear Science and Numerical Simulation, vol. 22, no. 1-3, pp. 92-94, 2015.

[41] J. Manafian and M. Lakestani, "A new analytical approach to solve some of the fractional-order partial differential equations," Indian Journal of Physics, vol. 91, no. 3, pp. 243-258, 2017.

[42] X. J. Yang, Advanced Local Fractional Calculus and its Applications, World Science Publisher, Singapore, 2012.

[43] X. J. Yang, "A short note on local fractional calculus of function of one variable," Journal of Applied Library and Information Science, vol. 1, no. 1, p. 113, 2012.

[44] X. J. Yang, "The zero-mass renormalization group differential equations and limit cycles in non-smooth initial value problems," Prespacetime Journal, vol. 3, no. 9, Article ID 913923, 2012.

[45] Z. Jia, M. Hu, Q. Chen, and S. Jai, "Local fractional differential equations by the Exp-function method," International Journal of Numerical Methods for Heat and Fluid Flow, vol. 25, no. 8, pp. 1845-1849, 2015.

[46] S. Sahoo and S. Saha Ray, "New solitary wave solutions of time-fractional coupled Jaulent-Miodek equation by using two reliable methods," Nonlinear Dynamics, vol. 85, no. 2, pp. 1167-1176, 2016.

[47] S. Sahoo and S. Saha Ray, "Solitary wave solutions for time fractional third order modified $\mathrm{KdV}$ equation using two reliable techniques $\left(\mathrm{G}^{\prime} / \mathrm{G}\right)$-expansion method and improved (G'/G)-expansion method," Physica A: Statistical Mechanics and Its Applications, vol. 448, pp. 265-282, 2016.

[48] J.-H. He, S. K. Elagan, and Z. B. Li, "Geometrical explanation of the fractional complex transform and derivative chain rule for fractional calculus," Physics Letters A, vol. 376, no. 4, pp. 257-259, 2012. 